\title{
éditionsOCDE
}

\section{AGRICULTURE, ALIMENTATION ET EMPLOI EN AFRIQUE DE L'OUEST}

\section{NOTES OUEST-AFRICAINES}

Avril 2018 No. 14 



\section{AGRICULTURE, ALIMENTATION ET EMPLOI EN AFRIQUE DE L'OUEST}

Cette note a été rédigée par

THOMAS ALLEN

PHILIPP HEINRIGS

INHOI HEO

Secrétariat du Club du Sahel et de l'Afrique de l'Ouest

Federal Ministry for Economic Cooperation and Development
Secrétariat du

CUO DU SAHEL ET DE 


\section{NOTES OUEST-AFRICAINES}

La série Notes ouest-africaines analyse les dynamiques socio-économiques, politiques et sécuritaires que traverse l'Afrique dans une perspective régionale et multidisciplinaire. Elle cherche à stimuler la discussion, rassembler les informations et mieux anticiper les transformations en cours pour les politiques à venir. La série vise à partager des études avec une large audience d'experts, de praticiens du développement, de décisionnaires et de lecteurs avertis. Les Notes sont disponibles en anglais et/ou en français ; les résumés dans les deux langues. Initiées par le Club du Sahel et de l'Afrique de l'Ouest (CSAO) pour éclairer les enjeux ouest-africains, ces analyses sont préparées par son Secrétariat, ses membres et partenaires, les autres départements de l'OCDE, des organisations internationales et autres experts et chercheurs.

Merci de citer cet ouvrage comme suit:

Allen, T., P. Heinrigs et I. Heo (2018), « Agriculture, alimentation et emploi en Afrique de l'Ouest ), Notes ouest-africaines, $N^{\circ} 14$, Éditions OCDE, Paris.

https://doi.org/10.1787/56d463a9-fr

Contact auteur : thomas.allen@oecd.org, philipp.heinrigs@oecd.org, inhoi.heo@oecd.org

ISSN 2415-1149

Les documents de travail de l'OCDE ne doivent pas être présentés comme exprimant les vues officielles de l'OCDE ou de ses pays membres. Les opinions exprimées et les arguments employés sont ceux des auteurs.

Ce document et toute carte qu'il peut comprendre ne préjugent en rien du statut de tout territoire, de la souveraineté s'exerçant sur ce dernier, du tracé des frontières et limites internationales, et du nom de tout territoire, ville ou région.

Les documents de travail exposent des résultats préliminaires ou des travaux de recherche en cours menés par l'auteur/les auteurs et sont publiés pour stimuler le débat sur un large éventail de questions sur lesquelles l'OCDE travaille. Les commentaires sur les documents de travail sont bienvenus et peuvent être adressés au Club du Sahel et de l'Afrique de l'Ouest, OCDE, 2 rue André-Pascal, 75775 Paris Cedex 16, France.

Autorisé pour publication par Laurent Bossard, Directeur, Secrétariat du Club du Sahel et de l'Afrique de l'Ouest (CSAO/OCDE).

Vous êtes autorisés à copier, télécharger ou imprimer du contenu OCDE pour votre utilisation personnelle. Vous pouvez inclure des extraits des publications, des bases de données et produits multimédia de l'OCDE dans vos documents, présentations, blogs, sites Internet et matériel d'enseignement, sous réserve de faire mention de la source OCDE et du copyright. Les demandes pour usage commercial ou de traduction devront être adressées à rights@oecd.org. 


\section{RÉSUMÉ}

L'économie alimentaire est le premier employeur en Afrique de l'Ouest, avec $66 \%$ de l'emploi total. Bien que la majorité de ces emplois soient encore dans l'agriculture, les activités non agricoles liées à l'alimentation progressent à mesure que le secteur s'adapte à l'évolution démographique, à l'urbanisation et à la croissance des revenus. Compte tenu du nombre d'emplois concernés, disposer d'une vision claire des perspectives de l'économie alimentaire est essentiel. Cette Note vise à quantifier et décrire la répartition de l'emploi dans les segments de l'agriculture, de la transformation, de la commercialisation et de la restauration hors domicile. Elle analyse les implications spatiales des transformations en cours, en particulier les interactions urbain-rural et la diversification de l'emploi rural. Enfin, elle propose des pistes politiques pour des stratégies d'emploi ciblées qui optimisent les synergies entre productivité agricole, emploi non agricole et interactions urbain-rural, et garantissent l'inclusion, en particulier des jeunes et des femmes.

Mots-clés : système alimentaire, emploi, jeunesse, femmes, chaînes de valeur

Classification : J21, J43, O11, Q13, Q18

\section{NOTE}

Ce travail a été réalisé dans le cadre du programme de travail et budget du Secrétariat du Club du Sahel et de l'Afrique de l'Ouest.

Il a bénéficié d'un financement additionnel du Ministère fédéral allemand de la Coopération économique et du Développement (BMZ).

\section{LE CLUB DU SAHEL ET DE L'AFRIQUE DE L'OUEST}

Le Club du Sahel et de l'Afrique de l'Ouest (CSAO) est une plateforme internationale indépendante. Son Secrétariat est hébergé au sein de l'Organisation de coopération et de développement économiques (OCDE).

\section{Mission}

La mission du CSAO est de promouvoir des politiques régionales à même d'améliorer le bien-être économique et social des populations ouest-africaines.

\section{Objectifs}

- Améliorer la gouvernance régionale de la sécurité alimentaire et nutritionnelle.

- Comprendre les transformations en cours dans la région et leurs implications en matière de politiques publiques par des analyses régionales, spatiales et prospectives.

\section{Membres}

Autriche, Belgique, Canada, Commission de la CEDEAO, CILSS, Commission de l'UEMOA, Union européenne, États-Unis, France, Luxembourg, Pays-Bas et Suisse.

La Banque mondiale, l'Agence de planification et de coordination du NEPAD et le Réseau des organisations paysannes et de producteurs agricoles sont observateurs.

Le CSAO a conclu des protocoles d'accord avec l'Agence du NEPAD et l'Université de Floride (Groupe de recherche sur le Sahel).

En savoir plus : http://www.oecd.org/fr/csao. 


\section{TABLE DES MATIÈRES}

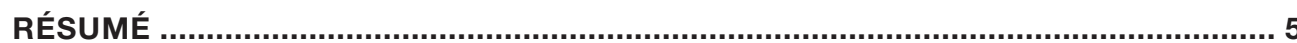

L'EMPLOI DANS L'ÉCONOMIE ALIMENTAIRE EN AFRIQUE DE L'OUEST.................. 7

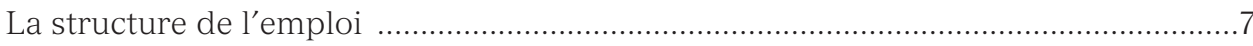

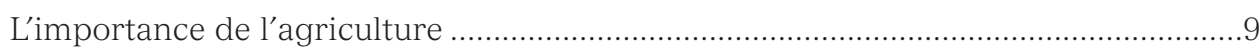

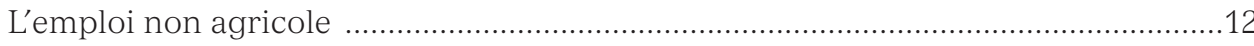

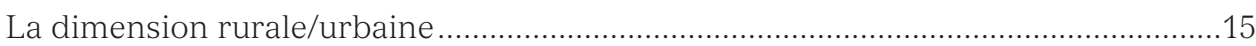

L'emploi des jeunes et la dimension du genre ……..................................................18

DIMENSIONS ÉCONOMIQUES ET TERRITORIALES DE L'EMPLOI DANS

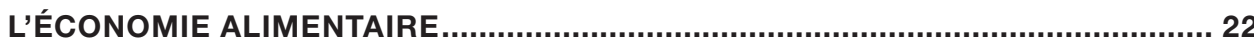

Chaînes de valeur alimentaires et emploi non agricole .............................................22

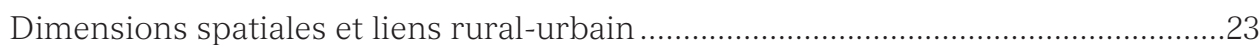

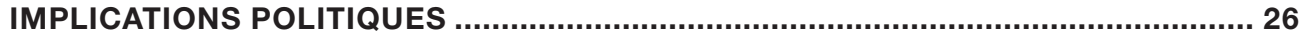

NOTES

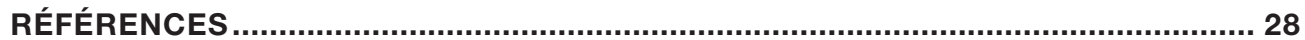

ANNEXE 1 : SOURCES DES DONNÉES ET STANDARDISATION................................ 30

ANNEXE 2 : MODÉLISATION DE L'EMPLOI - DIX PAYS ..........................................31 


\section{RÉSUMÉ}

-économie alimentaire est le premier employeur en Afrique de l'Ouest. Les diverses — activités, depuis la production agricole jusqu'à la transformation, au conditionnement, au transport, au stockage, à la distribution et au commerce de détail, représentent $66 \%$ de l'emploi total, soit 82 millions d'emplois, et sont à 78 \% dans l'agriculture. L'emploi non agricole dans les activités manufacturières et de services liées à l'alimentation augmente cependant en valeur absolue et relative.

L'urbanisation et la croissance des revenus sont à l'origine de transformations majeures de l'économie alimentaire. Les ménages s'approvisionnent de plus en plus sur les marchés, ils sont demandeurs d'une alimentation plus diversifiée, mais aussi plus facile à préparer et consommer. Ces évolutions touchent tous les pays et groupes de revenus, et entraînent un accroissement de la consommation de produits transformés et périssables à plus haute valeur ajoutée (Staatz et Hollinger, 2016 ; Allen et Heinrigs, 2016 ; Reardon et al., 2015). Ces changements dans les comportements de consommation et modes d'accès à l'alimentation entraînent le développement de nouvelles activités le long des chaînes de valeur alimentaires, notamment dans les segments non agricoles.

La demande de main-d'œuvre dans les activités associées à la transformation, à la commercialisation et à la restauration hors domicile est en augmentation. Ces activités entrent pour $22 \%$ dans l'emploi total de l'économie alimentaire et pour $31 \%$ dans le total des emplois non agricoles. Le segment de la commercialisation (transport, logistique, commerce de gros et de détail) représente la plus grande part de l'emploi non agricole de l'économie alimentaire (68 \%), suivi de la transformation (22\%). Compte tenu de la taille de l'économie alimentaire, ces activités jouent un rôle important dans l'emploi total. En Côte d'Ivoire, la transformation alimentaire est le plus gros contributeur à la valeur ajoutée du secteur formel et le deuxième plus gros contributeur à l'emploi formel (14 \% ; Hebous et Tran, 2017). Au Sénégal, les activités de transformation sont le premier sous-secteur manufacturier et ont progressé de $7.4 \%$ par an entre 2000 et 2010. La restauration hors domicile, qui rassemble l'alimentation de rue et la restauration, assure $10 \%$ de l'ensemble des emplois non agricoles de l'économie alimentaire, avec des parts nettement plus élevées dans certaines zones urbaines. Ce transfert de main-d'œuvre devrait s'amplifier à mesure que le système alimentaire, y compris l'agriculture, se spécialise et se diversifie, créant des opportunités d'emploi dans le secteur alimentaire domestique.

Ces transformations offrent des opportunités de création d'emploi dans les activités agricoles et non agricoles du système alimentaire, aussi bien en milieu rural qu'urbain. Ce potentiel est aujourd'hui insuffisamment exploité. Son développement exige une bonne compréhension du système alimentaire, en particulier des contextes locaux et de ses aspects spatiaux. Les stratégies d'emplois, notamment pour les jeunes et les femmes, doivent intégrer une approche systémique prenant en compte les liens entre productivité agricole, emploi non agricole et interactions urbain/rural. La création d'emploi non agricole dépendra du soutien apporté aux entreprises privées, en particulier aux petites et moyennes entreprises (PME), pour stimuler la croissance et l'emploi. Le soutien public au développement des chaînes de valeur et à l'acquisition des nouvelles compétences, dans une vision intégrée de l'économie alimentaire, ainsi que l'amélioration du climat des affaires, sont des éléments essentiels des stratégies de création d’emplois.

Les activités non agricoles et l'économie alimentaire en général jouent par ailleurs un rôle particulièrement important dans l'emploi des femmes : 68 \% des femmes ayant un emploi y travaillent. Elles occupent une part prépondérante des emplois dans les segments non agricoles, en particulier la restauration hors domicile, la transformation et la commercialisation. Pourtant, les femmes ont rarement accès aux ressources nécessaires pour développer leurs activités comme elles le pourraient et le souhaiteraient. Les politiques et investissements qui promeuvent les activités non agricoles de l'économie alimentaire profiteront en particulier aux femmes. 
Les emplois créés dans les chaînes de valeur alimentaires, y compris dans l'agriculture, exigent de nouvelles compétences. Anticiper ces besoins de formation et y répondre est essentiel. Pour créer et diversifier l'emploi, il faut doter les jeunes, notamment les jeunes femmes, des compétences et de la formation requises, et réduire les obstacles à l'accès aux ressources productives.

Cette Note vise à contribuer au débat en quantifiant et en décrivant la structure de l'emploi dans l'économie alimentaire en Afrique de l'Ouest, et en examinant les implications des transformations sur les dynamiques spatiales et territoriales.

\section{LA MÉTHODOLOGIE ET LES DONNÉES}

a production et la disponibilité de denrées alimentaires de bonne qualité en quantité L suffisante dépendent d'un nombre croissant d'activités et d'acteurs interdépendants. Ces activités vont de la production primaire, de la transformation et du transport à la commercialisation, de la restauration à l'alimentation de rue. Compte tenu de l'importance de l'économie alimentaire en Afrique de l'Ouest, la structure de l'emploi et ses évolutions ont des implications majeures sur la conception des stratégies. Les activités de l'économie alimentaire sont réparties dans différentes branches des comptes nationaux (secteurs primaire, secondaire et tertiaire), rendant difficile la compilation de données sur l'emploi (graphique 1). Les principales sources sont les données microéconomiques issues des études sur la mesure des niveaux de vie - Enquêtes intégrées sur l'agriculture de la Banque mondiale (LSMS-ISA) et enquêtes Emploi. Ces sources informent sur la répartition de la population active par activité ${ }^{1}$ et les temps de travail (nombre d'heures travaillées par personne). Dans la lignée de Tschirley, Kondo et Snyder (2017)², quatre segments sont identifiés ${ }^{3}$ :

Les principales sources proviennent des données microéconomiques issues des Études sur la mesure des niveaux de vie - Enquêtes intégrées sur l'agriculture de la Banque mondiale (LSMS-ISA) ainsi que des enquêtes Emploi. Ces sources informent sur la répartition de la population active par activité ${ }^{1}$ et les temps de travail (nombre d'heures travaillées par personne). Dans la lignée de Tschirley et al. (2017)², quatre segments sont identifiés ${ }^{3}$ :

1. l'agriculture, incluant l'emploi indépendant et l'emploi salarié dans les activités du secteur primaire consacrées à la production de produits agricoles pour la consommation humaine ;

2. la transformation alimentaire, couvrant tous les produits alimentaires et agricoles pour la consommation et les boissons ;

3. la commercialisation, à savoir toutes les activités de transport et de commerce de gros et de détail liées à l'alimentation ;

4. la restauration hors domicile, y compris l'alimentation de rue.

Les autres emplois relèvent de l'économie non alimentaire.

En l'Afrique de l'Ouest, les données LSMS ne sont disponibles que pour cinq pays (Burkina Faso, Ghana, Mali, Niger et Nigéria). Des enquêtes récentes sur la situation de l'emploi couvrent la Côte d’Ivoire (2012) et le Sénégal (2015). Ces sept pays représentent 82 \% de la population totale de l'Afrique de l'Ouest. Aucune donnée détaillée récente n'est accessible pour les dix autres pays. Pour ces derniers, les estimations s'appuient sur une modélisation macroéconomique à partir des données sectorielles agrégées de l’Organisation internationale du travail ${ }^{4}$ (OIT). 
Si les données issues des enquêtes LSMS et Emploi produisent des résultats robustes au regard des objectifs analytiques et du niveau d'agrégation de la Note, plusieurs limites doivent cependant être mentionnées. Le programme LSMS-ISA est une source majeure d'informations, notamment sur la main-d'œuvre agricole. Toutefois, le niveau d'agrégation des activités non agricoles dans les segments de la transformation, de la commercialisation et de la restauration hors domicile empêche une compréhension fine de la structure de l'emploi. De plus, la cohérence et la fiabilité des données recueillies restent préoccupantes. Les données sur l'utilisation du temps de travail, indispensables à l'estimation de la productivité et du sous-emploi lié à la durée du travail, montrent d'importantes variations d'un pays à l'autre. La même observation peut être faite pour les données sur l'inactivité, clés pour l'emploi des jeunes. Encore plus problématiques sont les divergences entre les données des variables, telles que les niveaux d'urbanisation, fondamentales pour la compréhension de l'évolution de l'emploi et des habitudes alimentaires. Selon LSMS, le taux d'urbanisation au Mali en 2014 est de 23 \% , alors qu'il est de 39 \% dans le rapport de l'Organisation des Nations Unies (ONU) sur les perspectives de l'urbanisation mondiale 5 (ONU, 2014). Pour le Nigéria, les pourcentages vont de 37 \% (LSMS) à 50 \% (ONU). Ces données démographiques sont utilisées pour calculer les coefficients de pondération des ménages et équilibrer l'échantillon afin qu'il soit représentatif de l'ensemble de la population, l'impact de cet écart sur la répartition des emplois dans l'économie alimentaire est donc important. La part de l'emploi non agricole augmenterait en effet de plus de $40 \%$ au Mali et de $12 \%$ Nigéria si des taux supérieurs d’urbanisation étaient utilisés.

Les statistiques disponibles ne sont pas suffisantes pour concevoir et suivre de manière opérationnelle les politiques et les programmes pour l'emploi. Il est en effet crucial de mieux comprendre la répartition de l'emploi au niveau microéconomique et la manière dont les décisions de production et de consommation sont reliées au sein du système alimentaire. Étant donné les caractéristiques territoriales et locales, des données spatiales sont également requises pour comprendre l'impact sur le marché du travail.

\section{L'EMPLOI DANS L'ÉCONOMIE ALIMENTAIRE EN AFRIQUE DE L'OUEST}

\section{La structure de l'emploi}

'économie alimentaire représente $66 \%$ de l'emploi total (graphique 1). La L plupart de ces emplois sont dans l'agriculture (78\%). Les emplois hors agriculture de l'économie alimentaire - transformation, commercialisation et restauration hors domicile - représentent $22 \%$. Au niveau régional, ils représentent $31 \%$ du total des emplois non agricoles. La transformation alimentaire entre pour $30 \%$ de l'emploi dans le secteur secondaire, tandis que la commercialisation et la restauration hors domicile comptent pour $31 \%$ dans de l'emploi du secteur tertiaire (graphique 1).

La part de l'emploi de l'économie alimentaire varie considérablement selon les pays. Au Tchad, au Niger, au Mali et au Burkina Faso, elle est de plus de 80 \% de l'emploi total, alors que dans certains pays côtiers (Gambie, Sénégal, Cabo Verde et Libéria), elle est inférieure à $50 \%$ (graphique 2). Ces écarts se retrouvent dans les différences entre les niveaux nationaux du produit intérieur brut (PIB) par habitant et entre les parts de l'agriculture dans l'emploi. Dans les pays où le niveau du PIB par habitant est plus faible et la part de l'agriculture dans l'emploi plus élevée, la part de la population active occupée travaillant dans l'économie alimentaire tend à être plus importante (tableau 1).

Cette situation reflète les processus de transformation structurelle, qui indiquent - conformément à la loi d’Engel - que plus le revenu augmente, plus la part de la consommation alimentaire dans le budget des ménages et la part de l'économie alimentaire dans le PIB et l'emploi diminuent. Toutefois, les différences entre pays sont également influencées par d'autres caractéristiques propres, comme les importations alimentaires, les exportations agricoles et le niveau d'urbanisation. 
Graphique 1

La structure de l'emploi en Afrique de l'Ouest

Emploi total en Afrique de l'Ouest par secteur
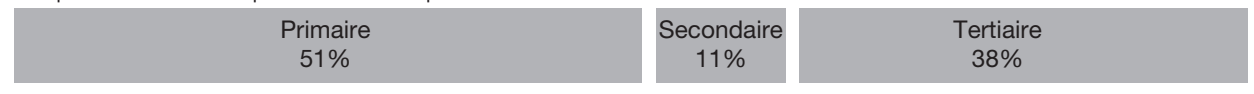

Parts de l'emploi relevant de l'économie alimentaire par secteur

\begin{tabular}{|c|c|c|}
\hline $\begin{array}{l}\text { Agriculture (pour l'alimentation) } \\
99 \%\end{array}$ & $70 \%$ & $69 \%$ \\
\hline Part de l'économie alimentaire dans l'emploi total & $\begin{array}{l}\text { Transformation } \\
\text { alimentaire } \\
30 \%\end{array}$ & $\begin{array}{l}\mathrm{n} \mid \begin{array}{c}\mathrm{R} \text { Restauration hors domicile } \\
4 \% \\
\text { Commercialisation } \\
27 \%\end{array} \\
\end{array}$ \\
\hline $\begin{array}{l}\text { Emploi dans l'économie alim€ } \\
66 \%\end{array}$ & & $\begin{array}{l}\text { Emploi hors économie alimentaire } \\
\qquad 34 \%\end{array}$ \\
\hline
\end{tabular}

Répartition de l'emploi dans l'économie alimentaire par segment

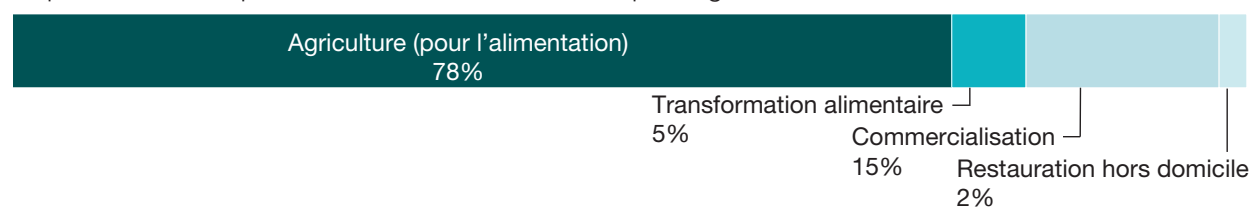

Sources : calculs des auteurs à partir de ANSD (2015), Enquête nationale sur l'emploi 2015 (base de données) ; Banque mondiale (2017a), Enquête sur la mesure des niveaux de vie (base de données) ; OIT (2017), Emploi par secteur (base de données) ; INS (2012), Enquête nationale sur la situation de l'emploi 2012, Côte d'Ivoire (base de données).

\section{Graphique 2}

Pourcentage et nombre de personnes exerçant un emploi dans l'économie alimentaire

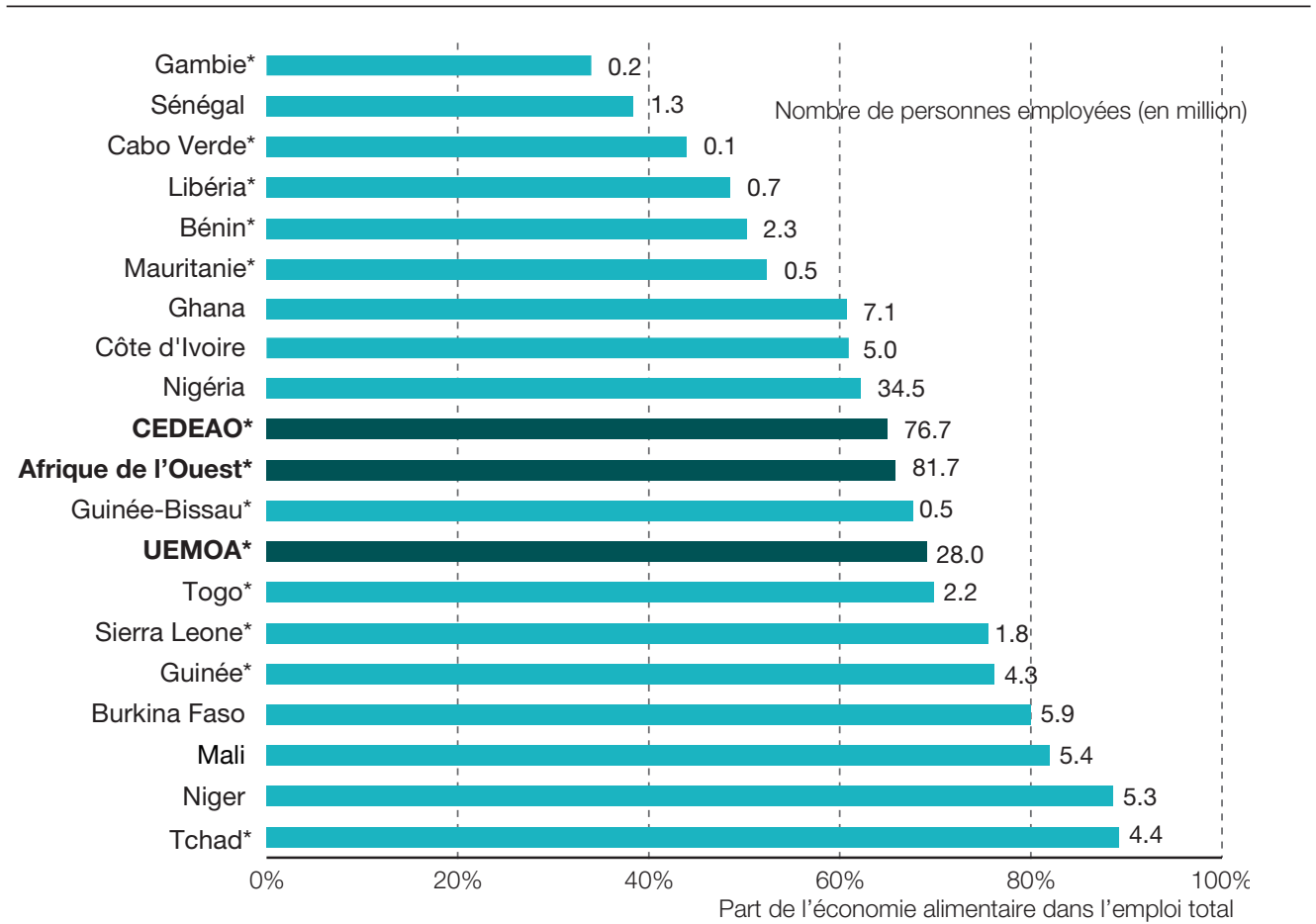

Notes : *données modélisées. Communauté économique des États de l'Afrique de l'Ouest (CEDEAO) ; Union économique et monétaire ouest-africaine (UEMOA).

Sources : calculs des auteurs à partir de ANSD (2015), Enquête nationale sur l'emploi 2015 (base de données) ; Banque mondiale (2017a), Enquête sur la mesure des niveaux de vie (base de données) ; OIT (2017), Emploi par secteur (base de données) ; INS (2012), Enquête nationale sur la situation de l'emploi 2012, Côte d'Ivoire (base de données). 
Tableau 1

Revenus, emploi, importations alimentaires et urbanisation

\begin{tabular}{|c|c|c|c|c|c|}
\hline & $\begin{array}{l}\text { PIB par habitant } \\
\text { (USD PPA) }\end{array}$ & $\begin{array}{l}\text { Emploi } \\
\text { agricole } \\
\text { (\% du total) }\end{array}$ & $\begin{array}{l}\text { Emploi économie } \\
\text { alimentaire } \\
\text { (\% du total) }\end{array}$ & $\begin{array}{l}\text { Importations } \\
\text { alimentaires } \\
\text { (\% du PIB) }\end{array}$ & $\begin{array}{l}\text { Niveau } \\
\text { d'urbanisation** }\end{array}$ \\
\hline Cabo Verde & 6302 & $28 \%$ & $45 \%{ }^{*}$ & $12 \%$ & $50 \%$ \\
\hline Nigéria & 5670 & $43 \%$ & $62 \%$ & $2 \%$ & $37 \%$ \\
\hline Ghana & 3940 & $44 \%$ & $61 \%$ & $6 \%$ & $50 \%$ \\
\hline Mauritanie & 3835 & $41 \%$ & $53 \% \%^{*}$ & $5 \%$ & $43 \%$ \\
\hline Côte d'Ivoire & 2762 & $52 \%$ & $61 \%$ & $7 \%$ & $42 \%$ \\
\hline Sénégal - - & 2445 & $32 \%$ & $39 \%$ & $9 \%$ & $51 \%$ \\
\hline Tchad & 2180 & $77 \%$ & $89 \% *$ & - & $28 \%$ \\
\hline Bénin & 2116 & $44 \%$ & $51 \%$ * & $12 \%$ & $48 \%$ \\
\hline Mali & 1965 & $75 \%$ & $82 \%$ & $4 \%$ & $23 \%$ \\
\hline Gambie & 1691 & $30 \%$ & $34 \%^{*}$ & $18 \%$ & $37 \%$ \\
\hline Burkina Faso & 1618 & $72 \%$ & $80 \%$ & $4 \%$ & $22 \%$ \\
\hline Guinée-Bissau & 1516 & $61 \%$ & $68 \% \%^{*}$ & - & $56 \%$ \\
\hline Togo & 1438 & $63 \%$ & $70 \% *$ & $9 \%$ & $50 \%$ \\
\hline Sierra Leone & 1401 & $68 \%$ & $76 \% \%^{*}$ & $7 \%$ & $37 \%$ \\
\hline Guinée & 1261 & $70 \%$ & $77 \%{ }^{\star}$ & $8 \%$ & $34 \%$ \\
\hline Niger & 948 & $81 \%$ & $89 \%$ & $7 \%$ & $16 \%$ \\
\hline Libéria & 836 & $45 \%$ & $49 \%^{*}$ & - & $42 \%$ \\
\hline
\end{tabular}

Notes: dollar américain parité de pouvoir d'achat (USD PPA) ; *données modélisées. **Données LSMS ou Enquêtes Emploi pour le Burkina Faso, la Côte d’Ivoire, le Ghana, le Mali, le Niger, le Nigéria et le Sénégal; Données Africapolis pour les 10 autres pays. Mise en forme conditionnelle : les cellules les plus colorées correspondent aux valeurs les plus élevées par colonne.

Sources : calculs des auteurs à partir de ANSD (2015), Enquête nationale sur l'emploi 2015 (base de données) ; Banque mondiale (2017a), Enquête sur la mesure des niveaux de vie (base de données) ; INS (2012), Enquête nationale sur la situation de l'emploi 2012, Côte d'Ivoire (base de données) ; OCDE/CSA0 (à paraître), "L' urbanisation des pays de l'Afrique de l'Ouest 1950-2015».

Au Burkina Faso, en Côte d'Ivoire, au Ghana et au Mali, l'emploi agricole dépend aussi de la taille des secteurs d'exportation (cacao, coton et caoutchouc). Au Mali et au Burkina Faso, la production de coton contribue fortement à l'emploi agricole, mais n'est pas liée aux modes de consommation alimentaire ${ }^{6}$. En Côte d'Ivoire et au Ghana, le secteur du cacao contribue sensiblement à l'emploi agroalimentaire, mais est tiré par la demande mondiale et non par les évolutions domestiques des revenus et de la consommation. En outre, les caractéristiques géographiques (Cabo Verde, Gambie), le niveau et la taille de la population urbaine, et l'importance des importations alimentaires influent sur la répartition globale de l'emploi (tableau 1). La structure de l'emploi dans l'économie alimentaire est principalement déterminée par la demande alimentaire domestique (Tschirley, Kondo et Snyder, 2016). Au niveau régional, la plus grande part de la consommation alimentaire est couverte par la production locale, les importations ne représentant que $8 \%$ du total des dépenses alimentaires ${ }^{7}$ (Allen et Heinrigs, 2016). Les importations et exportations alimentaires créent également des emplois. Les importations de produits non transformés, tels que les céréales, ou peu transformés génèrent des opportunités dans la transformation et la commercialisation. Les exportations favorisent l'emploi dans l'agriculture et, dans une moindre mesure, dans la transformation et la commercialisation (transports, stockage et logistique).

\section{L'importance de l'agriculture}

L'agriculture représente $78 \%$ des emplois du secteur alimentaire au niveau régional. Malgré des différences sensibles, tous les pays affichent une concentration dans l'agriculture d'au moins deux tiers des emplois de l'économie alimentaire (graphique 3). Cette part est étroitement liée aux systèmes agricoles et aux techniques de production : exploitations familiales, production à forte intensité de main-d'œuvre et exploitations de petite taille. Ces moyennes cachent cependant une forte hétérogénéité. En effet, dans tous les pays, de grandes exploitations mécanisées ou familiales de taille moyenne à forte productivité coexistent avec des structures plus traditionnelles. La relation entre le PIB 
Graphique 3

Structure de l'emploi dans l'économie alimentaire par secteur d'activité et comparaison en équivalents temps plein

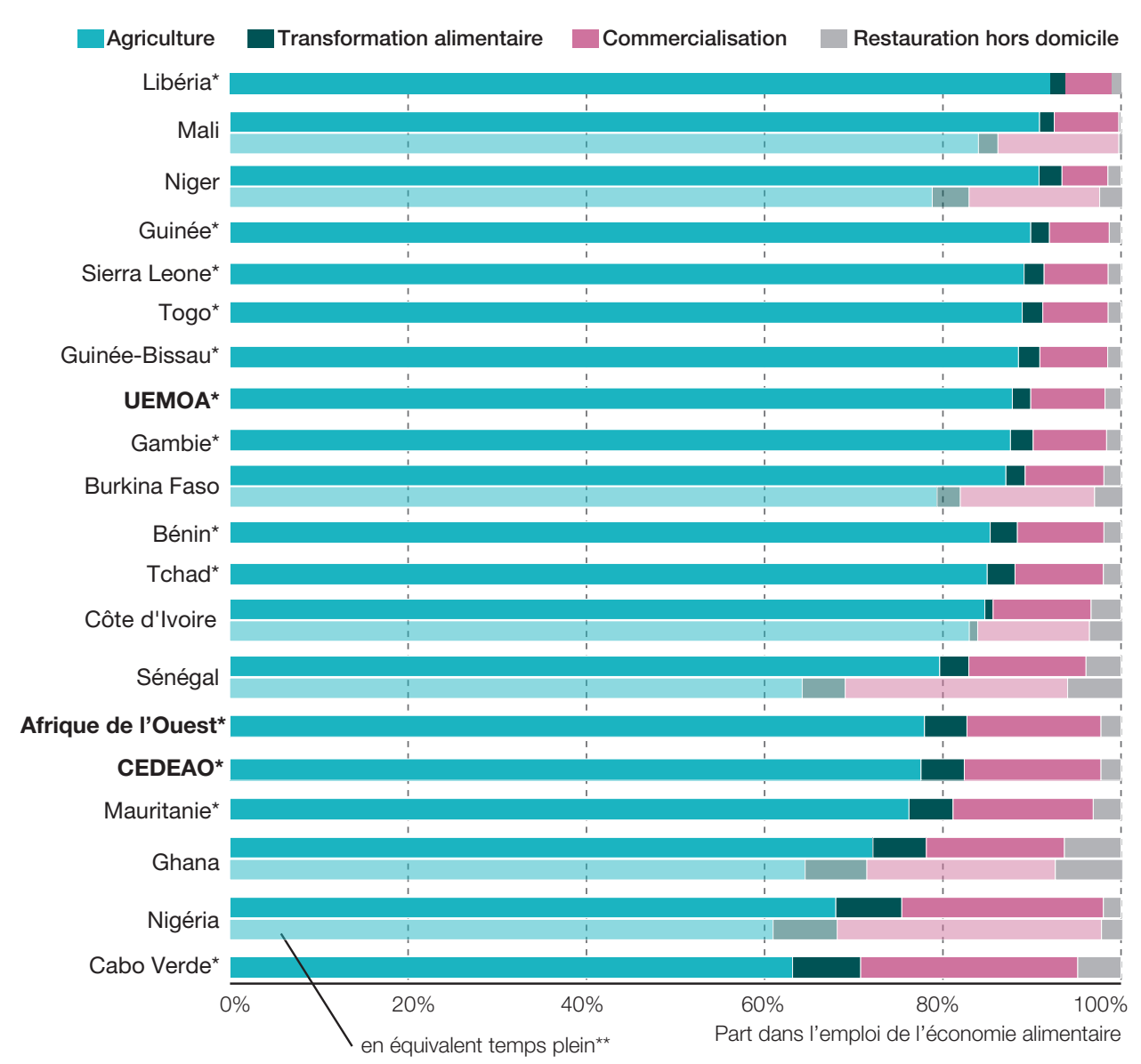

Note : * données modélisées.

Sources : calculs des auteurs à partir de ANSD (2015), Enquête nationale sur l'emploi 2015 (base de données) ; Banque mondiale (2017a), Enquête sur la mesure des niveaux de vie (base de données) ; INS (2012), Enquête nationale sur la situation de l'emploi 2012, Côte d'Ivoire (base de données), ; OIT (2017), Emploi par secteur (base de données).

par habitant et le pourcentage de travailleurs employés dans l'agriculture illustre aussi les interactions entre les évolutions des systèmes de production agricole, la demande d'emploi et les transformations structurelles. Le Cabo Verde, le Nigéria et le Ghana ont les PIB par habitant les plus élevés et les plus faibles pourcentages d'emplois dans l'agriculture (graphique 3). Le Niger, l'un des pays les plus pauvres, affiche l'une des plus fortes parts d'emplois dans l'agriculture. Entre ces deux extrêmes, la relation est moins claire, d'autres facteurs ayant une incidence sur la répartition de l'emploi.

Les différences de productivité peuvent influer sur la répartition de l'emploi. De plus en plus de sources confirment l'importance des écarts de productivité agricole entre et au sein des pays. L'un des moyens de mettre en évidence ces écarts et leur incidence sur l'emploi est de prendre en compte le nombre d'heures effectivement travaillées, c'est-à-dire d'estimer l'emploi en équivalents temps plein (ETP), et non plus seulement en fonction des 'activités déclarées (McCullough, 2017). Dans les sept pays pour lesquels des données sont disponibles, les employés agricoles déclarent nettement moins d’heures travaillées que ceux exerçant dans les segments aval de l'économie alimentaire. En moyenne, un agriculteur travaille 26 heures par semaine (première activité déclarée uniquement), contre 39 heures dans les secteurs aval. Ce sous-emploi lié à la durée du temps de travail, ou sous-emploi visible , s'explique par la saisonnalité de l'agriculture et/ou par la faible rémunération des 
surplus de production (et par conséquent du travail supplémentaire) engendrée par de faibles débouchés et un accès coûteux aux marchés (Christiaensen et al., 2017).

Ainsi, la répartition de l'emploi au Niger et au Burkina Faso en équivalents temps plein - et non l'activité déclarée - se traduit par une diminution sensible de la part de l'agriculture dans l'emploi de l'économie alimentaire, de $91 \%$ à $74 \%$ et de $87 \%$ à $75 \%$, respectivement (graphique 3). Le sous-emploi visible touche en particulier les activités agricoles, expliquant que l'on observe un doublement de la part de l'emploi non agricole dans l'économie alimentaire de ces pays. En Côte d'Ivoire, toutefois, la structuration de l'emploi demeure relativement stable, qu'elle soit exprimée en ETP ou sur la base des activités déclarées, ce qui indique que le sous-emploi en agriculture y est sensiblement inférieur. Des études confirment que ce moindre sous-emploi est liée à l'importance grandissante des cultures commerciales, au recours accru à de la main d'œuvre salariée, à la transformation structurelle de l'économie et à la forte urbanisation (Christiaensen et Lawin (2017). Le sous-emploi et les contraintes du travail saisonnier dans l'agriculture peuvent être réduits par des investissements dans les technologies, l'irrigation, la mécanisation ou la mise en place de systèmes agricoles mixtes (cultures et bétail) ainsi que par des investissements dans l'amélioration de l'accès aux marchés. Les données au Sénégal confirment le rôle des systèmes de production et de l'ouverture aux marchés dans l'amélioration de la productivité du travail en agriculture (voir encadré 1).

La répartition de l'emploi en ETP montre un recul de $10 \%$ de l'agriculture (7 points de pourcentage, de $75 \%$ à $68 \%$.) La part de l'emploi non agricole relevant de l'économie alimentaire augmente d'environ $30 \%$ (de $25 \%$ à $32 \%$ ). La part supérieure des activités non agricoles dans la structure de l'emploi en ETP est plus représentative des écarts de productivité du travail entre secteurs et de la répartition de la valeur ajoutée entre les segments. Les activités non agricoles représentent 40 \% de la valeur ajoutée totale de l'économie alimentaire en 2010 (Allen et Heinrigs, 2016).

Les différences de productivité peuvent influer sur la répartition de l'emploi. De plus en plus de sources confirment l'importance des écarts de productivité agricole entre et au sein des pays. L'un des moyens de mettre en évidence ces écarts et leur incidence sur l'emploi est de prendre en compte le nombre d'heures effectivement travaillées, c'est-à-dire d'estimer l'emploi en équivalents temps plein (ETP) ${ }^{8}$, et non plus seulement en fonction des activités déclarées (McCullough, 2017). Dans les sept pays pour lesquels des données sont disponibles, les employés agricoles déclarent nettement moins d’heures travaillées que ceux exerçant dans les segments aval de l'économie alimentaire. En moyenne, un agriculteur travaille 26 heures par semaine (première activité déclarée uniquement), contre 39 heures dans les secteurs aval. Ce sous-emploi lié à la durée du temps de travail, ou sous-emploi visible ${ }^{9}$, s'explique par la saisonnalité de l'agriculture et/ou par la faible rémunération des surplus de production (et par conséquent du travail supplémentaire) engendrée par de faibles débouchés et un accès coûteux aux marchés (Christiaensen et Premand, 2017).

Ainsi, la répartition de l'emploi au Niger et au Burkina Faso en ETP - et non l'activité déclarée - se traduit par une diminution sensible de la part de l'agriculture dans l'emploi de l'économie alimentaire, de $91 \%$ à 74 \% et de 87 \% à $75 \%$, respectivement (graphique 3). Le sous-emploi visible touche en particulier les activités agricoles, expliquant que l’on observe un doublement de la part de l'emploi non agricole dans l'économie alimentaire de ces pays. En Côte d'Ivoire, toutefois, la structuration de l'emploi demeure relativement stable, qu'elle soit exprimée en ETP ou sur la base des activités déclarées, ce qui indique que le sous-emploi en agriculture y est sensiblement inférieur. Des études confirment que ce moindre sous-emploi est lié à l'importance grandissante des cultures commerciales, au recours accru à de la main-d'œuvre salariée, à la transformation structurelle de l'économie et à la forte urbanisation (Christiaensen et Lawin, 2017). Le sous-emploi et les contraintes du travail saisonnier dans l'agriculture peuvent être réduits par des investissements dans les technologies, l'irrigation, la mécanisation ou la mise en place de systèmes agricoles 
mixtes (cultures et bétail), ainsi que par des investissements dans l'amélioration de l'accès aux marchés. Les données du Sénégal confirment le rôle des systèmes de production et de l'ouverture aux marchés dans l'amélioration de la productivité du travail en agriculture (voir encadré 1).

La répartition de l'emploi en ETP montre un recul de $10 \%$ de l'agriculture (7 points de pourcentage, de 75 \% à 68 \%.) La part de l'emploi non agricole relevant de l'économie alimentaire augmente d'environ $30 \%$ (de $25 \%$ à $32 \%$ ). La part supérieure des activités non agricoles dans la structure de l'emploi en ETP est plus représentative des écarts de productivité du travail entre secteurs et de la répartition de la valeur ajoutée entre les segments. Les activités non agricoles représentent $40 \%$ de la valeur ajoutée totale de l'économie alimentaire en 2010 (Allen et Heinrigs, 2016).

\section{L'emploi non agricole}

Les trois segments - transformation, commercialisation, restauration hors domicile représentent $22 \%$ de l'emploi total de l'économie alimentaire régionale. Bien que la répartition de l'emploi hors agriculture varie selon les pays, la commercialisation est clairement le principal segment non agricole de l'économie alimentaire. En revanche, il ne se dégage pas de répartition uniforme de l'emploi entre la transformation et la restauration hors domicile par pays. La commercialisation représente $15 \%$ de l'emploi total dans l'économie alimentaire, suivie de la transformation (5\%) et de la restauration hors domicile (2\%) (tableau 2). Ensemble, ces secteurs entrent pour $31 \%$ dans l'emploi non agricole total régional.

Tableau 2

Part de l'emploi non agricole dans l'emploi total de l'économie alimentaire

\begin{tabular}{|c|c|c|c|c|}
\hline & $\begin{array}{l}\text { Emploi hors agriculture } \\
\text { dans l'éco. alimentaire } \\
(1+2+3)\end{array}$ & $\begin{array}{l}\text { Transformation } \\
\text { alimentaire } \\
\text { (1) }\end{array}$ & $\begin{array}{l}\text { Commercialisation } \\
\text { (2) }\end{array}$ & $\begin{array}{l}\text { Restauration } \\
\text { hors domicile } \\
\text { (3) }\end{array}$ \\
\hline Cabo Verde* & $37 \%$ & $8 \%$ & $25 \%$ & $5 \%$ \\
\hline Nigéria & $32 \%$ & $7 \%$ & $23 \%$ & $2 \%$ \\
\hline Ghana & $28 \%$ & $6 \%$ & $16 \%$ & $6 \%$ \\
\hline Mauritanie ${ }^{\star}$ & $24 \%$ & $5 \%$ & $16 \%$ & $3 \%$ \\
\hline Afrique de l'Ouest* & $22 \%$ & $5 \%$ & $15 \%$ & $2 \%$ \\
\hline Côte d'Ivoire & $20 \%$ & $1 \%$ & $16 \%$ & $3 \%$ \\
\hline Sénégal - & $18 \%$ & $3 \%$ & $11 \%$ & $4 \%$ \\
\hline Tchad $^{*}$ & $15 \%$ & $3 \%$ & $10 \%$ & $2 \%$ \\
\hline Bénin* & $15 \%$ & $3 \%$ & $10 \%$ & $2 \%$ \\
\hline Burkina Faso & $13 \%$ & $2 \%$ & $9 \%$ & $2 \%$ \\
\hline Gambie* $^{*}$ & $13 \%$ & $3 \%$ & $8 \%$ & $2 \%$ \\
\hline Guinée-Bissau* & $12 \%$ & $2 \%$ & $8 \%$ & $2 \%$ \\
\hline Togo* & $11 \%$ & $2 \%$ & $7 \%$ & $2 \%$ \\
\hline Sierra Leone* & $11 \%$ & $2 \%$ & $7 \%$ & $1 \%$ \\
\hline Guinée* & $10 \%$ & $2 \%$ & $7 \%$ & $1 \%$ \\
\hline Niger & $9 \%$ & $3 \%$ & $5 \%$ & $1 \%$ \\
\hline Mali & $9 \%$ & $2 \%$ & $7 \%$ & $0 \%$ \\
\hline Libéria* & $8 \%$ & $2 \%$ & $5 \%$ & $1 \%$ \\
\hline
\end{tabular}

Note : * données modélisées. Mise en forme conditionnelle : les cellules les plus colorées correspondent aux valeurs les plus élevées par colonne.

Sources : calculs des auteurs à partir de ANSD (2015), Enquête nationale sur l'emploi 2015 (base de données) ; Banque mondiale (2017a), Enquête sur la mesure des niveaux de vie (base de données) ; INS (2012), Enquête nationale sur la situation de l'emploi 2012, Côte d'Ivoire (base de données) ; OIT (2017), Emploi par secteur (base de données). 
Le développement des segments non agricoles de l'économie alimentaire est étroitement corrélé aux changements des habitudes alimentaires des ménages. Des études récentes en Afrique de l'Ouest confirment des évolutions des comportements de consommation conformes à la loi de Bennett (Allen et Heinrigs, 2016 ; Staatz et Hollinger, 2016). Elles montrent qu'avec l'augmentation du revenu et l'urbanisation, la consommation de produits frais et périssables, comme les fruits et légumes, de produits laitiers, de viandes et d'aliments transformés progresse rapidement. Les produits alimentaires périssables et transformés impliquent une plus grande création de valeur ajoutée en aval de l'agriculture (chaîne du froid, traitement et conditionnement). Ces évolutions se traduisent par un déplacement plus marqué de l'emploi vers les segments non agricoles de l'économie alimentaire. En outre, la croissance de la commercialisation est étroitement corrélée au développement des marchés.

\section{La transformation alimentaire}

La transformation est le premier sous-secteur manufacturier en termes d'emploi dans la région. Bien qu'elle ne représente que $5 \%$ de l'emploi dans l'économie alimentaire, sa part s'élève à $30 \%$ dans l'emploi total du secteur secondaire. Au Niger et au Nigéria, elle représente près de 50 \% des activités manufacturières (graphique 4). Une grande part de ces emplois concerne des entreprises artisanales et des PME du secteur informel. Une étude récente de la Banque mondiale, analysant l'emploi dans le secteur formel ivoirien, montre cependant que ( la transformation alimentaire (CITI 15) est le plus important contributeur à la valeur ajoutée et le deuxième en termes d'emploi ) (Hebous et Tran, 2017). « Si la part des entreprises de l'agribusiness n’était que de 4 \% en 2012, celles-ci génèrent $18 \%$ des emplois ) (ibid).

\section{Graphique 4}

Part de la transformation alimentaire dans l'emploi manufacturier total (2012-15)

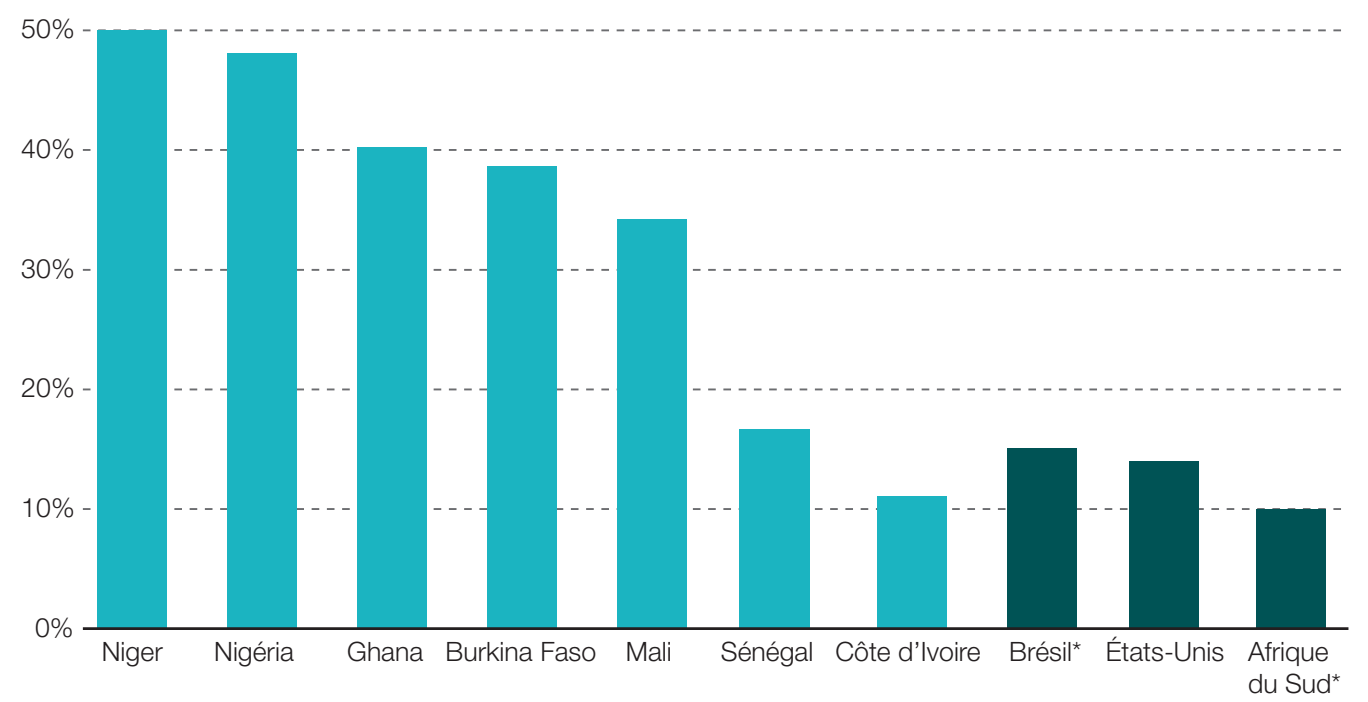

Note: *2008

Sources : calculs des auteurs à partir de ANSD (2015), Enquête nationale sur l'emploi 2015 (base de données) ; Banque mondiale (2017a), Enquête sur la mesure des niveaux de vie (base de données) ; INS (2012), Enquête nationale sur la situation de l'emploi 2012, Côte d'Ivoire (base de données) ; Nesheim, Oria et Yih (dir. pub.) (2015), "A Framework for Assessing Effects of the Food System" ; OIT (2012a), Sectoral country profil - Brazil et OIT (2012b), Sectoral country profil - South Africa. 
Du point de vue de l'emploi, la transformation alimentaire présente trois caractéristiques importantes. Premièrement, les agro-industries (par exemple les minoteries, les brasseries et les entreprises de transformation) sont plus souvent situées en dehors des villes principales que les autres secteurs industriels, dans les petites villes et les zones rurales (Henderson et McNamara, 2000 ; Christiaensen et Lawin, 2017). Deuxièmement, elles créent des liens solides en amont et en aval avec les autres activités du système alimentaire et non alimentaire. La transformation est un débouché de plus en plus important pour la production agricole, ce qui contribue à stabiliser la demande. Ces deux caractéristiques peuvent avoir des effets importants sur l'emploi dans les économies locales. Les activités de transformation sont dominées par les femmes et tendent à être peu qualifiées et à forte intensité de main-d'œuvre, offrant davantage d'inclusion.

Troisièmement, la demande pour les activités de transformation devrait continuer de croître à moyen terme. Au Nigéria, sur la base des projections de la consommation, l'emploi devrait augmenter de 13 \% sur les 5 prochaines années (Tschirley, Kondo et Snyder, 2016). Dans une perspective de création d'emplois, la croissance dans ce secteur dépendra avant tout de la capacité des entreprises locales de transformation et d'agribusiness à s'approvisionner auprès des producteurs locaux (Staatz et Hollinger, 2016).

\section{La commercialisation alimentaire}

La commercialisation alimentaire - transports, stockage, commerce de gros et de détail - représente plus de $70 \%$ du total des emplois de l'économie alimentaire non agricole et 27 \% des emplois dans le secteur des services. Leur développement est lié à l'urbanisation et à la dépendance des ménages aux marchés pour leur alimentation. Les ménages ruraux dépendent aussi de plus en plus des marchés pour l'accès à l'alimentation. Les Burkinabè et le Nigérians dépensent plus de 60 \% de leur budget alimentaire sur les marchés, dont $36 \%$ pour des produits transformés ${ }^{10}$. En outre, l'évolution de la demande alimentaire et la réduction des coûts de transport se traduisent par une augmentation de la demande de denrées produites dans des régions toujours plus éloignées (Tacoli et Agergaard, 2017).

La part de la commercialisation dans l'emploi est la plus importante dans les pays plus urbanisés, comme le Sénégal, le Ghana, la Côte d'Ivoire et le Nigéria, et la plus faible au Niger et au Mali, plus ruraux (tableau 2). Ces types d'activités se développeront et fourniront le plus grand nombre d'emplois non agricoles dans les années à venir.

\section{La restauration hors domicile}

Elle représente $2 \%$ des emplois de l'économie alimentaire (10 \% des emplois non agricoles). Cette part varie considérablement d'un pays à l'autre. Au Sénégal et au Ghana, elle représente $6 \%$ et $4 \%$, respectivement, contre $1 \%$ et moins au Niger et au Mali (tableau 2). Ces chiffres reflètent les écarts dans les dépenses des ménages. Dans les villes principales, la restauration hors domicile représente une part importante mais variable des dépenses alimentaires, allant de plus de $30 \%$ à Abidjan, Cotonou et Lomé à moins de 10 \% à Bamako, Conakry et Freetown (Bricas, Tchamda et Mouton, 2016). Ces différences s'expliquent par des facteurs culturels, mais aussi par la taille, la configuration des villes, le réseau de transport et les horaires de travail.

La restauration hors domicile est étroitement associée aux revenus et devrait augmenter plus rapidement que les autres segments de l'industrie alimentaire (Staatz et Hollinger, 2016). En outre, elle génère une forte valeur ajoutée (également sur les produits importés) et contribue à la demande pour les autres segments du secteur ces activités (Tschirley, Kondo et Snyder, 2016). Elle est également cruciale pour l'emploi des femmes (voir section suivante). 


\section{La dimension rurale/urbaine ${ }^{11}$}

On relève une répartition spatiale claire des activités de l'économie alimentaire. Celles agricoles sont essentiellement rurales, alors que les activités de commercialisation et de restauration hors domicile, qui dépendent de la proximité et de la taille des bassins de consommation, sont urbaines. La transformation se répartit de façon plus homogène entre zones urbaines et rurales, et se situe généralement à proximité, soit de la demande finale, soit des zones de production des intrants.

\section{Graphique 5}

La répartition spatiale de l'emploi par segment

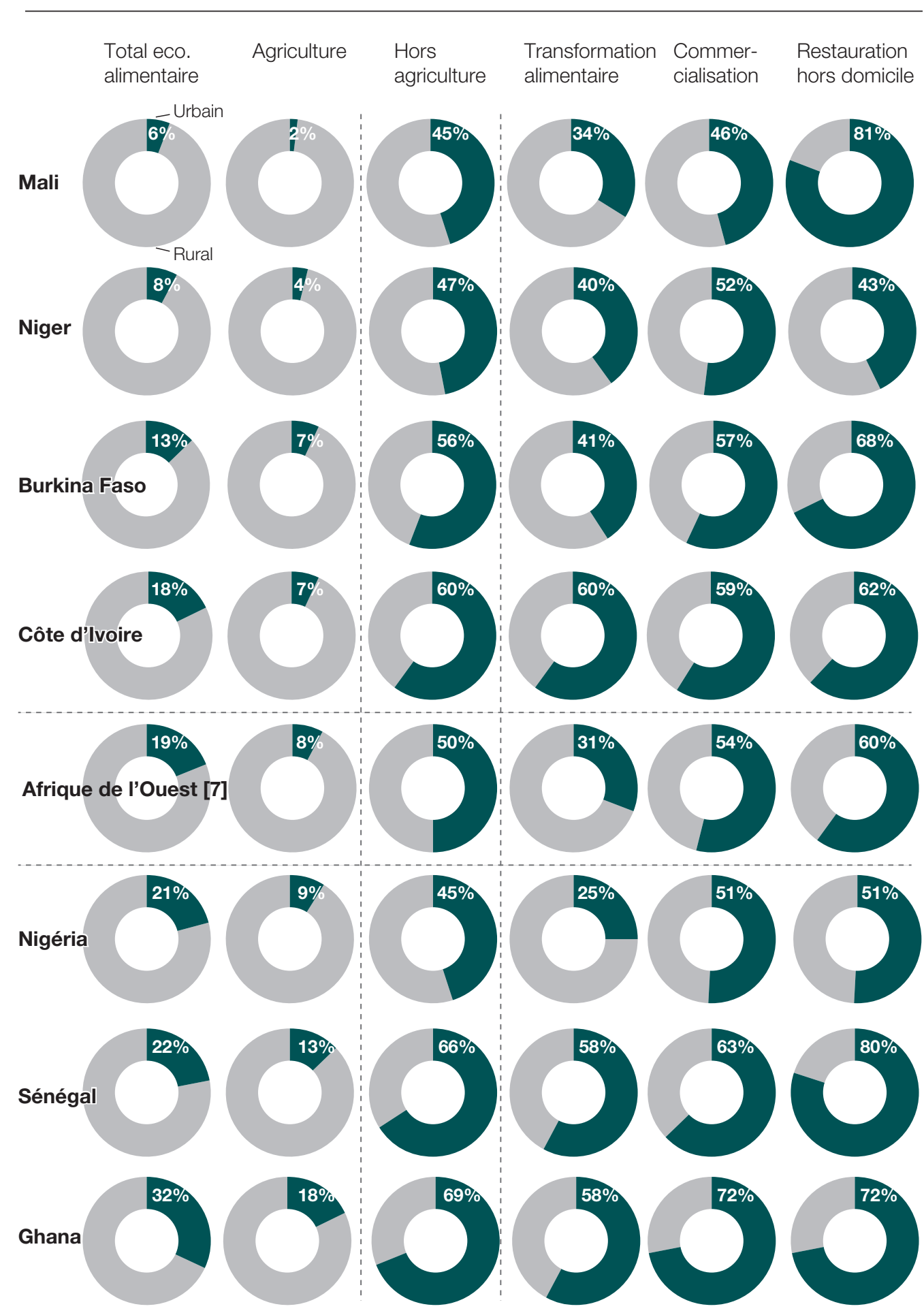

Sources : calculs des auteurs à partir de ANSD (2015), Enquête nationale sur l'emploi 2015 (base de données) ; Banque mondiale (2017a), Enquête sur la mesure des niveaux de vie (base de données) ; INS (2012), Enquête nationale sur la situation de l'emploi 2012, Côte d'Ivoire (base de données). 


\section{L'emploi dans l'économie alimentaire en milieu rural}

L'économie alimentaire représente $81 \%$ de l'emploi rural total (graphique 7). Cette part s'explique par la prédominance des activités agricoles, qui représentent 85 \% des emplois de l'économie alimentaire en milieu rural. L'analyse de l'emploi rural hors agriculture de l'économie alimentaire montre que les segments non agricoles offrent des opportunités intéressantes de diversification de l'emploi et qu'il existe d'importantes différences entre et au sein des pays (carte 1).

La commercialisation représente $10 \%$ de l'ensemble des emplois ruraux de l'économie alimentaire, suivie par la transformation (4\%) et la restauration hors domicile $(1 \%$; graphique 6). Les pays où la structure de l'emploi dans l'économie alimentaire est plus variée (avec une part plus importante des activités non agricoles) tendent aussi à avoir un emploi rural plus diversifié dans les trois segments non agricoles (transformation, commercialisation et restauration hors domicile). Au Nigéria, la transformation et la commercialisation représentent respectivement $7 \%$ et $14 \%$ de l'emploi rural total de l'économie alimentaire. Ces pourcentages sont de $2 \%$ et $3 \%$ au Niger (graphique 6). En Côte d'Ivoire, l'emploi rural dans la transformation est particulièrement faible par rapport à la taille du secteur alimentaire non agricole.

Graphique 6

Diversification de l'emploi rural de l'économie alimentaire

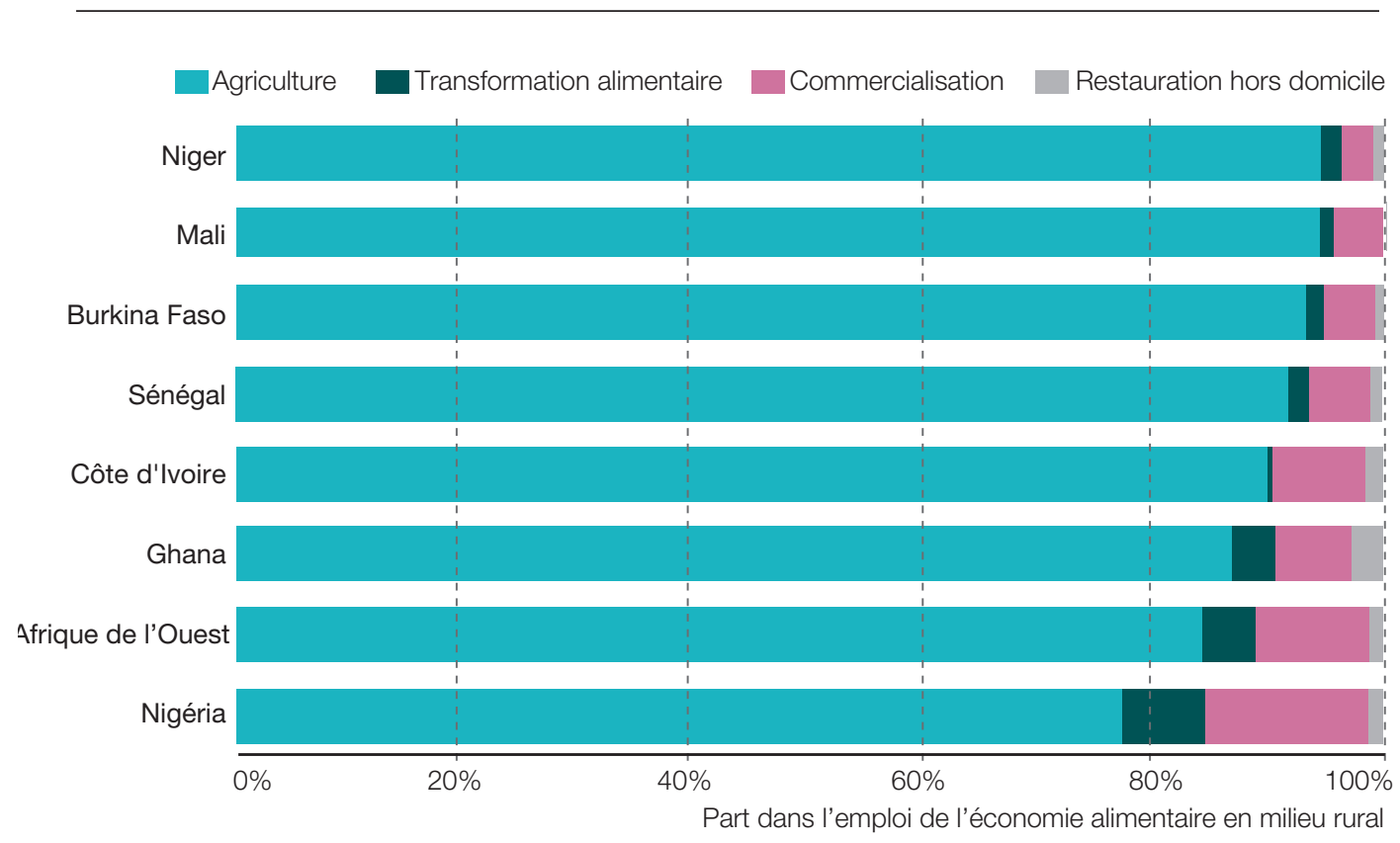

Sources : calculs des auteurs à partir de ANSD (2015), Enquête nationale sur l'emploi 2015 (base de données) ; Banque mondiale (2017a), Enquête sur la mesure des niveaux de vie (base de données) ; INS (2012), Enquête nationale sur la situation de l'emploi 2012, Côte d'Ivoire (base de données).

La diversification de l'emploi en milieu rural reflète les transformations plus larges de l'économie alimentaire. L'offre d'emploi agricole s'accroît dans la mesure où la production de produits laitiers, de viande, de poisson, de fruits et légumes est plus intensive en maind'œuvre, ce qui compense les réductions dans les cultures vivrières de base. L'offre dans les segments non agricoles de l'économie alimentaire augmente en raison de la croissance des activités associées au stockage, au transport, au traitement, au conditionnement et au commerce de gros et de détail (Christiaensen et Premand, 2017). Le développement des activités non agricoles en zones rurales dépend aussi de la diversification de la demande sur les marchés ruraux, y compris de la demande de restauration hors domicile (qui stimule l'emploi dans les segments non agricoles). 
La transformation et la commercialisation sont indispensables au développement des chaînes de valeur alimentaires. Leurs faibles parts dans l'emploi rural masquent leur rôle clé dans la croissance agricole, la génération de revenus et la transformation rurale. Ces activités sont les points d'entrée sur les marchés, liant la production agricole et la consommation alimentaire. Le calibrage, le stockage et le commerce de gros permettent aux entreprises de transformation d'accéder aux matières premières ; le conditionnement des produits est nécessaire à leur conservation et à leur transport, alors que la logistique et le commerce de gros sont indispensables pour atteindre le consommateur final. Le développement de ces activités non agricoles interdépendantes favorise aussi la production et l'intensification (Proctor et Berdegué, 2016 ; Jayne, Chamberlin et Headey, 2014).

\section{L'emploi dans l'économie alimentaire en milieu urbain}

Les emplois de l'économie alimentaire représentent 35 \% de l'emploi urbain total (graphique 7), dont 57 \% pour la commercialisation et la restauration hors domicile. Ces activités dépendent de la taille des marchés alimentaires et varient entre les pays. Au Ghana, au Sénégal et en Côte d'Ivoire, 66 \% des emplois dans la commercialisation et la restauration hors domicile se situent en zones urbaines, contre 52 \% au Mali, au Niger et au Burkina Faso.

Ils concernent les petits commerces, la vente à l'étalage, les marchands ambulants ou l'alimentation de rue. Ces activités - surtout informelles - jouent un rôle central dans le système de commercialisation en zones urbaines. Les ménages urbains vulnérables en sont dépendants pour leur approvisionnement. Du fait des coûts de préparation et du manque d'équipements à domicile, ils achètent de petites quantités quotidiennement à des vendeurs ambulants (Proctor et Berdegué, 2016).

Graphique 7

Part de l'économie alimentaire dans l'emploi total par milieu

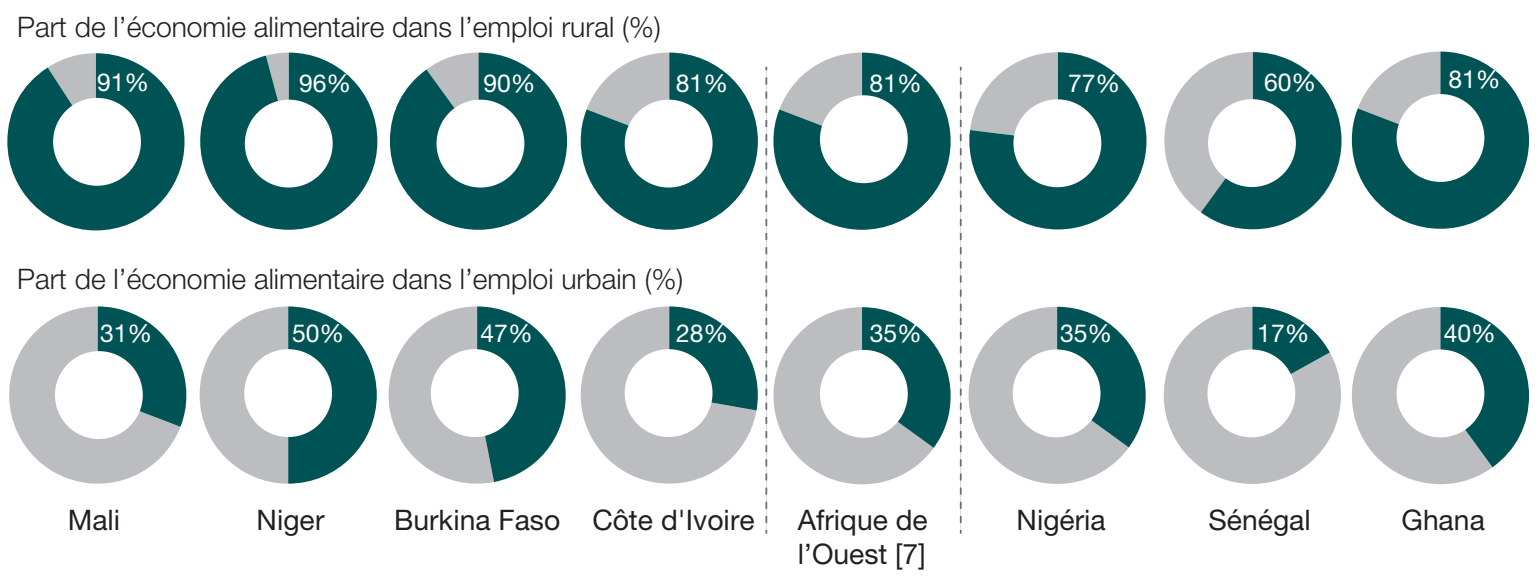

Sources : calculs des auteurs à partir de ANSD (2015), Enquête nationale sur l'emploi 2015 (base de données) ; Banque mondiale (2017a), Enquête sur la mesure des niveaux de vie (base de données) ; INS (2012), Enquête nationale sur la situation de l'emploi 2012, Côte d'Ivoire (base de données).

L'agriculture représente $34 \%$ des emplois de l'économie alimentaire en milieu urbain. Au Nigéria et au Niger (seuls pays pour lesquels on dispose de données géolocalisées détaillées), la majorité des producteurs urbains sont situés dans les villes petites et moyennes. Les activités concernent la production sur de petites parcelles de denrées périssables à forte valeur ajoutée, comme les légumes, ce qui permet aux producteurs de surmonter les contraintes foncières et de bénéficier de la proximité des marchés. Dans certaines villes, les facteurs géographiques expliquent pour une large part le pourcentage élevé d'activités agricoles. C'est le cas de la pêche dans les villes côtières. Dans le district densément peuplé d'Ablekuma Sud, à Accra (Ghana), 18 \% des ménages pratiquent la pêche, deuxième activité après la préparation du kenkey (plat à base de maïs fermenté) 
et la transformation du poisson (23 \% ; Ministère de l'Alimentation et de l'Agriculture du Ghana, 2016). Toutefois, la part des activités agricoles en zones urbaines est probablement gonflée par la prise en compte de ménages vivant en ville, mais menant des activités agricoles en dehors.

\section{L'emploi des jeunes et la dimension du genre}

\section{Diversification de l'emploi des jeunes}

Les jeunes occupent une place importante dans les priorités en matière d'emploi. En Afrique de l'Ouest, $19 \%$ de la population a entre 15 et 24 ans, soit 36 \% de l'ensemble de la population en âge de travailler (15-65 ans), contre $26 \%$ pour la tranche des 25-34 ans.

Dans l'ensemble, les jeunes ont le plus faible taux d'activité, avec de grandes différences nationales, entre les zones rurales et urbaines, et selon le genre (tableau 3). Les taux d'activité sont sensiblement plus faibles dans les pays côtiers (entre 33 et 57 \%) que dans les pays sans littoral - Niger, Mali et Burkina Faso (64 à 82 \%). Ces pourcentages élevés sont influencés par la forte participation des jeunes au marché du travail dans les zones rurales, conjuguée à une forte part de la population rurale dans la population totale. Dans l'ensemble des pays, le taux de participation des jeunes est plus élevé dans les milieux ruraux qu'urbains, notamment celui des jeunes hommes. La seule exception est la Côte d'Ivoire, où Le taux d'activité des jeunes femmes dans les zones urbaines est supérieur à celui des hommes.

Tableau 3

Taux d'activité des jeunes par pays, milieu et sexe

\begin{tabular}{|c|c|c|c|c|c|c|}
\hline & \multirow{3}{*}{$\begin{array}{l}\text { Jeunes en } \\
\text { age de travailler' } \\
(15-24)\end{array}$} & \multicolumn{4}{|c|}{ Jeunes (15-24) } & \multirow{3}{*}{$\begin{array}{l}\text { Adultes en age } \\
\text { de travailler } \\
(25-65)\end{array}$} \\
\hline & & \multicolumn{2}{|c|}{ Urbain } & \multicolumn{2}{|c|}{ Rural } & \\
\hline & & Hommes & Femmes & Hommes & Femmes & \\
\hline Burkina Faso & $82 \%$ & $55 \%$ & $52 \%$ & $96 \%$ & $92 \%$ & $93 \%$ \\
\hline Ghana & $71 \%$ & $32 \%$ & $26 \%$ & $89 \%$ & $83 \%$ & $83 \%$ \\
\hline Màli & $64 \%$ & $46 \%$ & $18 \%$ & $86 \%$ & $64 \%$ & $81 \%$ \\
\hline Afrique de l'Ouest & $57 \%$ & $44 \%$ & $42 \%$ & $74 \%$ & $71 \%$ & $92 \%$ \\
\hline Nigeria & $53 \%$ & $35 \%$ & $42 \%$ & $72 \%$ & $64 \%$ & $82 \%$ \\
\hline Côte d'Ivoire & $46 \%$ & $32 \%$ & $31 \%$ & $60 \%$ & $49 \%$ & $83 \%$ \\
\hline Niger & $36 \%$ & $27 \%$ & $26 \%$ & $48 \%$ & $35 \%$ & $82 \%$ \\
\hline Senegal & $33 \%$ & $34 \%$ & $20 \%$ & $52 \%$ & $27 \%$ & $58 \%$ \\
\hline
\end{tabular}

Note : mise en forme conditionnelle : les cellules les plus colorées correspondent aux valeurs les plus élevées par colonne. Première colonne conditionnelle par colonne ; colonnes 2, 3, 4 et 5 conditionnelles par ligne.

Sources : calculs des auteurs à partir de ANSD (2015), Enquête nationale sur l'emploi 2015 (base de données) ; Banque mondiale (2017a), Enquête sur la mesure des niveaux de vie (base de données) ; INS (2012), Enquête nationale sur la situation de l'emploi 2012, Côte d'Ivoire (base de données).

La majorité des jeunes inactifs sont scolarisés (73 \%). Là encore, le lieu de résidence (77 \% des urbains) et le sexe jouent un rôle important. Un plus grand nombre de jeunes hommes (78 \%) sont scolarisés que de jeunes femmes (55\%). Trente pour cent des jeunes femmes déclarées économiquement inactives restent chez elles et ce pourcentage tend à être plus élevé en zones rurales (42 \%). En outre, l'inactivité économique et le chômage tendent à être plus élevés parmi les groupes les plus aisés, les individus les plus pauvres ne pouvant être inactifs ou au chômage (Christiaensen et Premand, 2017). Les taux d'inactivité économique chutent rapidement avec l'âge : 61 \% des 15-19 ans, contre seulement 30 \% des 25-29 ans. Globalement, 73 \% des jeunes (15-24 ans) occupant un emploi vivent en zones rurales et 60 \% sont des hommes. Au Mali, au Niger et au Burkina Faso, ce pourcentage est toutefois beaucoup plus élevé (90\%, $87 \%$ et $80 \%$, respectivement), avec aussi une forte part de l'emploi agricole (63\%) dans l'emploi total des jeunes (graphique 8). 


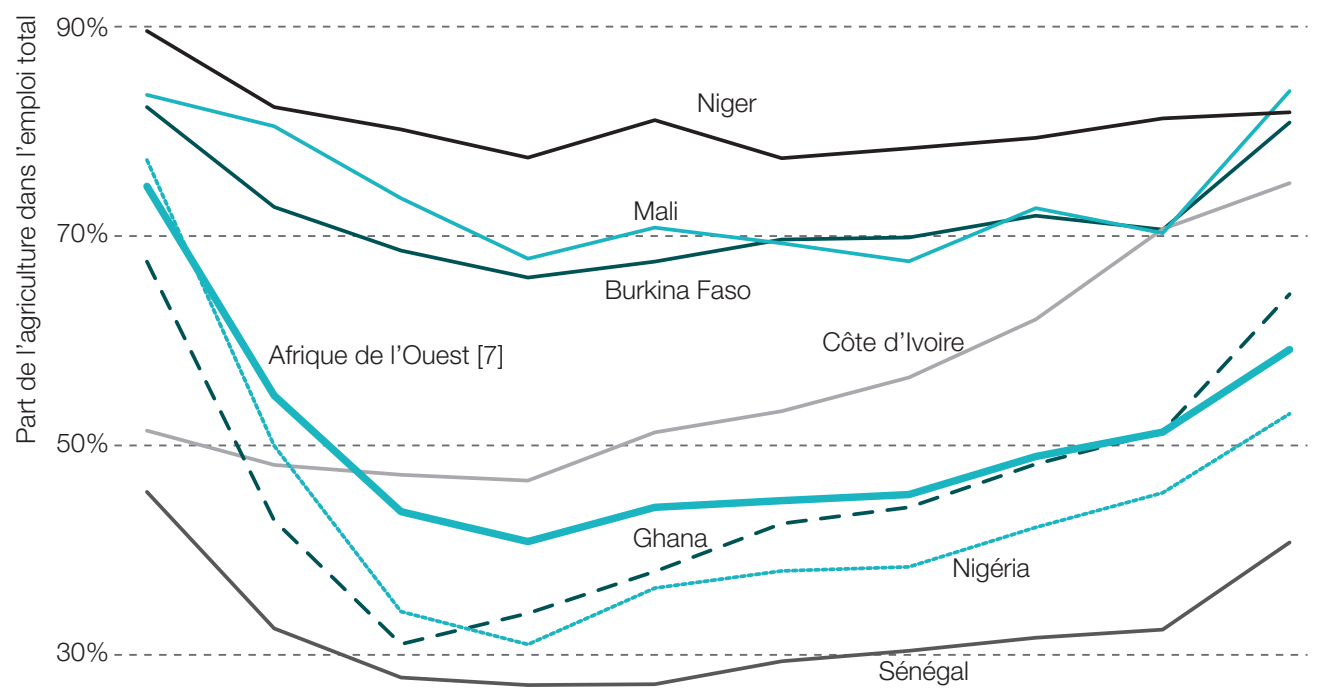

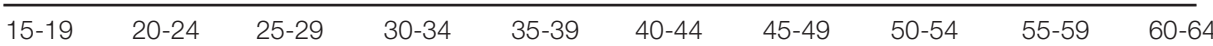

Sources : calculs des auteurs à partir de ANSD (2015), Enquête nationale sur l'emploi 2015 (base de données) ; Banque mondiale (2017a), Enquête sur la mesure des niveaux de vie (base de données) ; INS (2012), Enquête nationale sur la situation de l'emploi 2012, Côte d'Ivoire (base de données).

Les jeunes entrant sur le marché du travail rejoignent essentiellement les secteurs non agricoles. Les 30-34 ans travaillent proportionnellement moins dans le domaine de l'agriculture que les autres tranches d'âge (graphique 8). Cette configuration est marquée dans les pays où les jeunes inactifs sont nombreux et les taux de scolarisation plus élevés. Ainsi, au Nigéria, 71 \% des actifs de 15 à 19 ans travaillent dans l'agriculture, contre $31 \%$ pour les 30-34 ans. Bien que la situation soit moins prononcée au Burkina Faso, au Mali et au Niger, on y relève les mêmes tendances. Au Burkina Faso, l'agriculture représente 80 \% de l'emploi total des 15-19 ans, mais seulement 64 \% pour les 30-34 ans. Les faibles obstacles à l'entrée, en particulier dans les exploitations familiales, et l'absence d'opportunités dans d'autres secteurs expliquent sans doute la forte présence des jeunes actifs de 15-19 ans dans l'agriculture (McCullough, 2017).

La baisse de la part de l'emploi dans l'agriculture s'accompagne d'une augmentation de l'emploi dans les activités non agricoles du système alimentaire et dans les activités hors alimentation (graphique 9). En Côte d’Ivoire, au Ghana, au Nigéria et au Sénégal, la part de l'emploi hors agriculture passe de 31 \% pour les 15-19 ans à 67 \% pour les 30-34 ans. Pour les mêmes tranches d'âge au Burkina Faso, au Mali et au Niger, elle est passée de $17 \%$ à $31 \%$. La transition des jeunes vers des activités non agricoles a lieu plus rapidement et en plus grand nombre que dans les générations plus âgées, en partie parce que les jeunes ont une meilleure formation et plus de mobilité (Christiaensen, 2017). En outre, les difficultés d'accès aux moyens de production et l'absence de perspectives sont autant de facteurs qui poussent les jeunes hors de l'agriculture.

La structure de l'emploi des 20-24 ans dans l'économie alimentaire reflète les évolutions de la population en âge de travailler. Les jeunes peuvent cependant jouer un rôle important dans le développement des activités alimentaires, notamment en agriculture. Du fait des transformations dans ce secteur, un niveau plus élevé de formation et de compétences est nécessaire pour profiter des opportunités d'emploi et développer de nouvelles activités (Jayne, Chamberlin et Headey, 2014). Au Sénégal, les données montrent que les jeunes à la tête d'exploitations agricoles sont plus fortement impliqués dans les organisations 
Graphique 9

La transition des jeunes vers le marché du travail

a) Côte d'Ivoire, Ghana, Nigéria, Sénégal

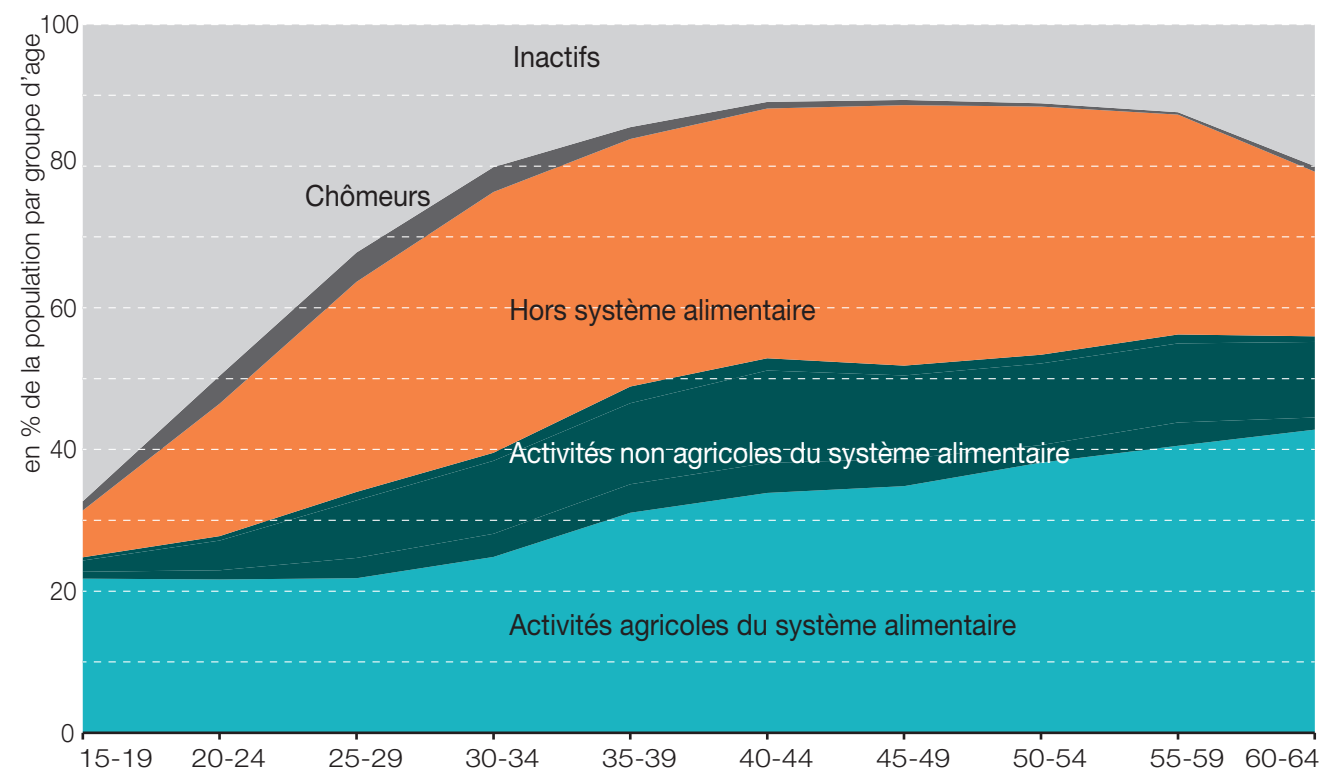

b) Burkina Faso, Mali, Niger

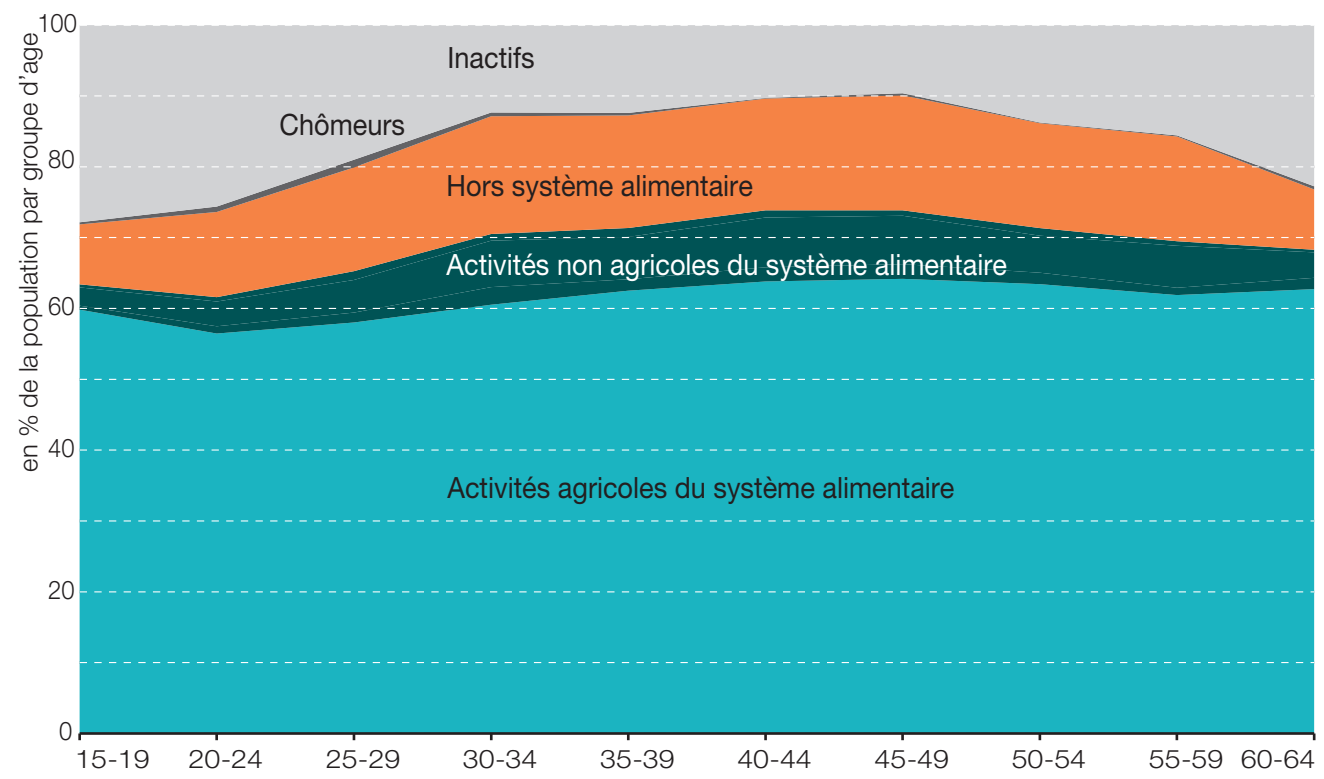

Sources : calculs des auteurs à partir de ANSD (2015), Enquête nationale sur l'emploi 2015 (base de données) ; Banque mondiale (2017a), Enquête sur la mesure des niveaux de vie (base de données) ; INS (2012), Enquête nationale sur la situation de l'emploi 2012, Côte d'Ivoire (base de données).

de producteurs et les coopératives agricoles, ainsi que dans les associations de crédit et d'usagers de l'eau (IPAR, 2015). Le soutien à la création et à la diversification de l'emploi exige de doter les jeunes des compétences et de la formation requises et de réduire les obstacles à l'accès aux moyens de production, plus élevés pour les jeunes, notamment les jeunes femmes. 


\section{L'emploi dans l'économie alimentaire et les femmes}

L'économie alimentaire joue un rôle important pour l'emploi féminin : 68 \% des femmes ayant un emploi y travaillent. Bien qu'elles ne représentent qu'un pourcentage légèrement plus élevé du total de l'emploi dans le système alimentaire (51\%), elles dominent dans les segments non agricoles. Elles représentent $88 \%$ du personnel de la restauration hors domicile, $83 \%$ dans la transformation et $71 \%$ dans la commercialisation (graphique 10). Quelque 37 \% des femmes travaillant dans l'économie alimentaire sont employées dans les segments non agricoles, contre 11 \% des hommes.

\section{Graphique 10}

Part de l'emploi des femmes par segment

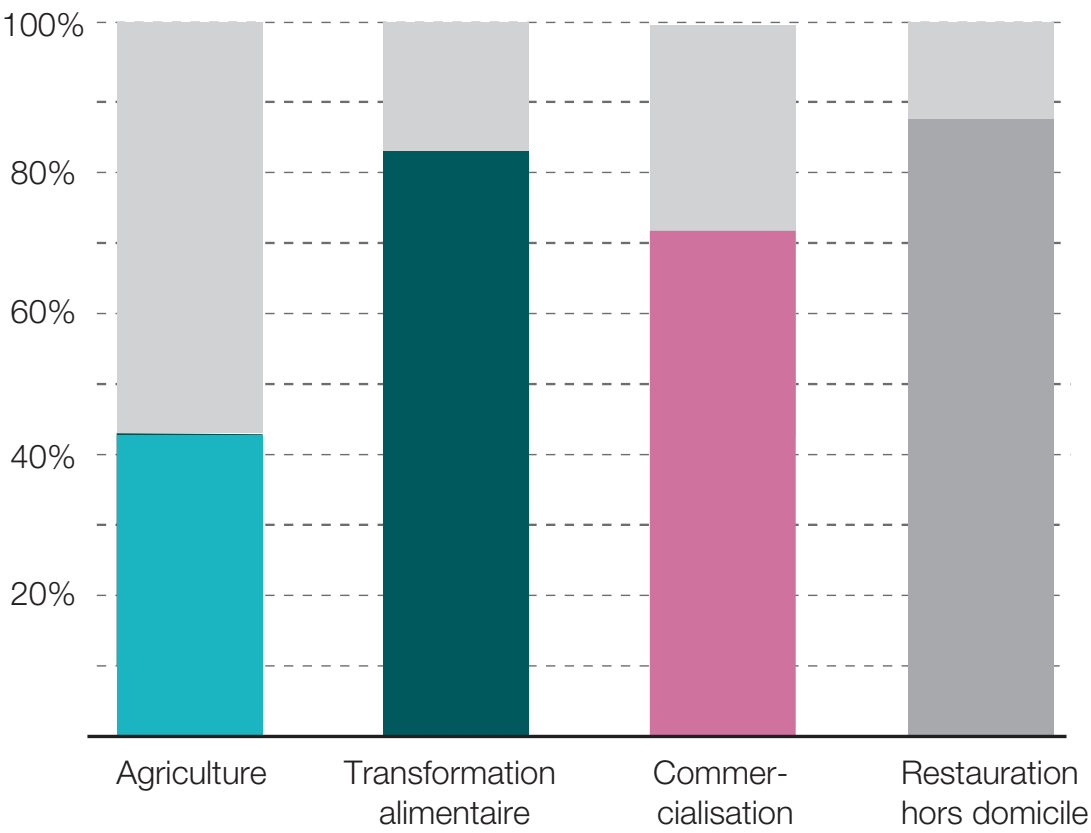

Sources : calculs des auteurs à partir de ANSD (2015), Enquête nationale sur l'emploi 2015 (base de données) ; Banque mondiale (2017a), Enquête sur la mesure des niveaux de vie (base de données) ; INS (2012), Enquête nationale sur la situation de l'emploi 2012, Côte d'Ivoire (base de données).

En zones rurales, $75 \%$ des femmes employées dans l'économie alimentaire travaillent dans l'agriculture. Elles ont cependant nettement moins de chances que les hommes d'être cheffes d'exploitation, un moindre accès aux meilleures terres (en termes de qualité des sols et d'équipements comme les systèmes d'irrigation), et ont généralement de plus petites exploitations (IPAR, 2015). Les différences de genre en matière d'accès aux moyens de production et les normes et perceptions sociales qui encouragent ou découragent la participation des femmes dans certaines activités conduisent ces dernières à se diriger vers des activités de niche où les barrières à l'entrée sont moins rigides (IPAR, 2015). La transformation alimentaire et la restauration hors domicile sont presque exclusivement féminines dans les zones rurales. Les femmes ont quatre fois plus de probabilité de travailler dans les segments non agricoles en zones rurales.

Dans les zones urbaines, les activités alimentaires non agricoles représentent un emploi sur trois chez les femmes (un sur sept chez les hommes). Cette proportion est plus élevée au Niger (un emploi sur deux), au Burkina Faso et au Ghana. Ces activités peuvent être particulièrement rentables. En milieu urbain, la transformation et la restauration hors domicile sont lucratives et en plein essor. Dans le district d'Attécoubé à Abidjan (Côte d'Ivoire), 20 \% de la population travaille dans la fabrication de l'attiéké. Les productrices gagnent en moyenne 30 à 50 \% de plus que les autres professions représentées dans le quartier (coiffeurs, mécaniciens, tapissiers) et 85 \% d'entre elles appartiennent aux groupes à revenus élevés ou moyens (MADR/Côte d'Ivoire, 2015). 
Tableau 4

Part de l'emploi des femmes dans l'économie alimentaire

\begin{tabular}{|c|c|c|c|c|c|}
\hline & $\begin{array}{l}\text { Total économie } \\
\text { alimentaire }\end{array}$ & Agriculture & $\begin{array}{l}\text { Transformation } \\
\text { alimentaire }\end{array}$ & $\begin{array}{l}\text { Commer- } \\
\text { cialisation }\end{array}$ & $\begin{array}{l}\text { Restauration } \\
\text { hors domicile }\end{array}$ \\
\hline Ghana & $58 \%$ & $49 \%$ & $77 \%$ & $84 \%$ & $92 \%$ \\
\hline Burkina Faso & $56 \%$ & $54 \%$ & $79 \%$ & $71 \%$ & $82 \%$ \\
\hline Afrique de l'Ouest (7) & $51 \%$ & $43 \%$ & $83 \%$ & $72 \%$ & $88 \%$ \\
\hline Mali & $50 \%$ & $49 \%$ & $62 \%$ & $66 \%$ & $51 \%$ \\
\hline Nigéria & $50 \%$ & $37 \%$ & $87 \%$ & $72 \%$ & $92 \%$ \\
\hline Niger & $49 \%$ & $47 \%$ & $84 \%$ & $59 \%$ & $93 \%$ \\
\hline Cote d'ivoire & $48 \%$ & $43 \%$ & $30 \%$ & $71 \%$ & $71 \%$ \\
\hline Sénégal & $37 \%$ & $36 \%$ & $25 \%$ & $33 \%$ & $77 \%$ \\
\hline
\end{tabular}

Note : mise en forme conditionnelle : les cellules les plus colorées correspondent aux valeurs les plus élevées par colonne. Première colonne conditionnelle par colonne ; colonnes 2, 3, 4 et 5 conditionnelles par ligne.

Sources : calculs des auteurs à partir de ANSD (2015), Enquête nationale sur l'emploi 2015 (base de données) ; Banque mondiale (2017a), Enquête sur la mesure des niveaux de vie (base de données) ; INS (2012), Enquête nationale sur la situation de l'emploi 2012, Côte d'Ivoire (base de données).

Les femmes ont rarement accès aux ressources nécessaires pour développer leurs activités. Les institutions du secteur privé, comme les banques, sont mal informées du potentiel des PME agroalimentaires et ne leur allouent pas de crédits. Les politiques et les stratégies publiques accordent trop peu d'attention et de ressources à ces segments, malgré leur importance pour le développement de l'économie alimentaire (Staatz et al., 2016). Les conditions et les obstacles à la participation économique des femmes varient entre et au sein des pays, en fonction des filières, des réseaux commerciaux, des freins à la mobilité, etc. (Bouchama et al., 2018). Toutefois, des politiques et des investissements intégrant les contraintes spécifiques au genre et encourageant les segments non agricoles de l'économie alimentaire pourraient avoir un impact fort sur les activités économiques féminines et le développement des systèmes alimentaires.

\section{DIMENSIONS ÉCONOMIQUES ET TERRITORIALES DE L'EMPLOI DANS L'ÉCONOMIE ALIMENTAIRE}

I es évolutions des systèmes alimentaires créent de nouvelles opportunités dans les L activités agricoles et non agricoles, dans les zones rurales et urbaines. Les zones rurales en bénéficieront tout particulièrement. Les pays qui ont transformé avec succès leur agriculture et leur économie rurale, comme le Viet Nam, sont ceux où la réduction de la pauvreté a été la plus forte, conformément à la loi de Bennett (Christiaensen etPremand, 2017). Il importe toutefois de bien comprendre que les liens entre les activités de l'économie alimentaire (chaînes de valeur), qui assurent la connexion entre production et consommation, reflètent également les liens entre zones rurales et urbaines. Ces interactions sont des éléments importants du développement de l'économie alimentaire et de la transformation rurale et doivent être mieux appréhendées pour promouvoir la création d'emploi.

\section{Chaînes de valeur alimentaires et emploi non agricole}

Les chaînes de valeur alimentaires participent du développement d'économies rurales diversifiées et y représentent une part importante de l'offre d'emploi non agricole. Les transformations des habitudes alimentaires, qui influent sur les décisions de production de denrées riches en protéines (produits laitiers, viande) et de fruits et légumes à plus forte valeur ajoutée, et donc les types d'emplois en agriculture, stimulent également l'offre d'emploi non agricole dans les activités aval des chaînes de valeur, comme le stockage, le transport, la transformation, le conditionnement, le commerce de gros et de détail (Reardon, 2015). 
L'augmentation de la productivité agricole sera indispensable pour développer ces opportunités. L'agriculture fournira de nombreux emplois dans l'économie alimentaire au cours des prochaines décennies (Tschirley, Kondo et Snyder, 2016 ; encadré 1). Des analyses récentes (Christiaensen et Premand, 2017 ; Jayne, Chamberlin et Headey, 2014 ; IPAR, 2015) montrent que l'amélioration de la productivité et l'augmentation des revenus agricoles figurent parmi les principaux facteurs de transition de l'emploi rural et de stimulation d’une économie rurale non agricole.

La majorité des nouveaux emplois non agricoles dans les chaînes de valeur alimentaires sont liés à l'agriculture. Un secteur agricole productif est indispensable pour assurer leur compétitivité et favoriser la demande d'activités rurales non agricoles. Celles-ci se situent principalement dans la transformation (notamment les premières étapes comme le nettoyage, le calibrage et l'emballage) et la commercialisation (stockage, transport et commerce de gros et de détail). L'existence et l'accès aux infrastructures, comme les marchés, les installations de stockage et logistiques, l'électricité et les routes, sont essentielles. Un climat des affaires propice au développement de l'agrobusiness est tout aussi important. Ceci implique notamment un fonctionnement efficace des marchés pour l'accès aux moyens de production, comme la terre et les crédits, des acteurs bien organisés le long des filières, l'accès à l'information (sur les débouchés, les prix, les niveaux de qualité et les normes), des services de vulgarisation et la disponibilité d'une main-d'œuvre qualifiée. L'emplacement géographique et la proximité des marchés stimulent également la demande en services connexes et intrants, comme les revendeurs d'équipements agricoles et d'outillage, ou les fournisseurs de semences, stimulant encore la création d'emplois et l'économie locale (Proctor et Berdegué, 2016). Compte tenu des projections de la demande alimentaire, le potentiel de création d'emplois dans l'économie alimentaire est important. Il importe de le développer de manière concertée pour répondre au défi de l'emploi en Afrique.

Ces opportunités exigent de nouvelles compétences : l'utilisation de nouvelles techniques (semences, engrais, pratiques de conservation) ; la maîtrise des technologies de l'information et de la communication (TIC) pour accéder aux informations sur les marchés ; mais également une meilleure compréhension du fonctionnement des acteurs publics et privés ; ainsi que le recours à des mécanismes de financement, de conseil et de commercialisation (Jayne, Chamberlin et Headey, 2014). Anticiper ces besoins en formation et y répondre sont des aspects essentiels de l'action à mener en faveur de l'emploi.

\section{Dimensions spatiales et liens rural-urbain}

Les transformations en cours dans le système alimentaire révèlent également l'importance des dynamiques spatiales, en particulier des interactions entre zones rurales et urbaines. Celles-ci se sont intensifiées avec l'urbanisation et le rôle croissant des marchés dans l'accès à l'alimentation. Ces interactions spatiales - qui incluent la circulation des biens et personnes - déterminent le nombre et la diversité des acteurs et activités impliqués dans les chaînes de valeur alimentaires, ce qui a une incidence sur l'emploi domestique. Ces dynamiques, observables dans la plupart des pays du monde, ne sont pas statiques et évoluent avec le développement, l'urbanisation et les progrès en matière de connexion (Berdegué et al., 2014 ; Prieto Curiel, Heinrigs et Heo, 2017). 
Encadré 1

Productivité agricole et offre d'emploi dans les segments de l'économie alimentaire au Sénégal

Le potentiel que représente l'emploi dans l'agriculture est sous-estimé dans les récents débats. Cette situation s'explique par une sous-estimation de la demande alimentaire domestique et de la capacité des producteurs locaux à approvisionner les marchés, conjuguée à une surestimation des importations (Allen et Heinrigs, 2016). Un autre argument avancé est celui de la faible productivité du travail dans l'agriculture et, par conséquent, de l'absence de création d'emplois rémunérateurs. Or, des études récentes (McCullough, 2017 et Christiaensen et Premand, 2017) amènent à penser que les écarts de productivité entre l'agriculture et les activités secondaires et tertiaires sont nettement plus faibles qu'on ne le suppose (plutôt de l'ordre de 1:2 que de 1:6). En outre, la productivité du travail dans l'agriculture est très hétérogène, soulignant le potentiel d'accroissement de la productivité intra-sectorielle sous les conditions agro-écologiques existantes. Les données de la Côte d'Ivoire montrent que la productivité moyenne du travail dans l'agriculture est la plus élevée à Abidjan et dans d'autres zones urbaines (Christiaensen et Lawin, 2017). Cette hétérogénéité témoigne de l'importance des facteurs spatiaux et du contexte local.

Elle tient pour partie au sous-emploi. Les données du Sénégal (IPAR, 2015) comparant trois zones rurales montrent l'impact de l'utilisation des technologies et de l'ouverture aux marchés sur la productivité et l'offre d'emploi (graphique 11). Le graphique fait apparaître des écarts importants entre trois zones, caractérisées chacune par des systèmes de production différents. La productivité du travail dans la région des Niayes, dominée par l'horticulture à destination des marchés urbains, est 17 fois plus élevée que dans le Bassin arachidier, où se pratique la culture pluviale de l'arachide et du mil. Les rendements y sont 12 fois supérieurs. Pourtant, pendant la saison des pluies, les écarts de productivité du travail entre le Bassin arachidier et la région des Niayes ne sont plus que de un pour trois. Ceux-ci sont fortement influencés par les différences dans les niveaux d'activité. L'étude de l'IPAR explique que les taux d'activité « varient fortement selon les périodes de l'année (hivernage, contre-saison). Le Bassin arachidier se singularise par des taux d'activités élevés en hivernage et bas en saison sèche. Cela n'est guère surprenant eu égard au système de production pluvial en vigueur. Les Niayes affichent des taux d'activités relativement élevés dans les deux saisons » (IPAR, 2015). La hausse de la productivité, stimulée par des investissements dans des systèmes de production plus spécialisés et axés sur la commercialisation, accroît également l'offre d'emploi dans l'agriculture. C'est ce que l'on observe dans la région du Delta avec une culture intensive de céréales et de légumes (riz, oignon et tomate), reposant sur l'irrigation à grande échelle et la mécanisation. Deux fois plus d'exploitations embauchent de la main-d'œuvre dans la région du Delta que dans les Niayes.

\section{Graphique 11}

Productivité agricole et offre d'emploi au Sénégal

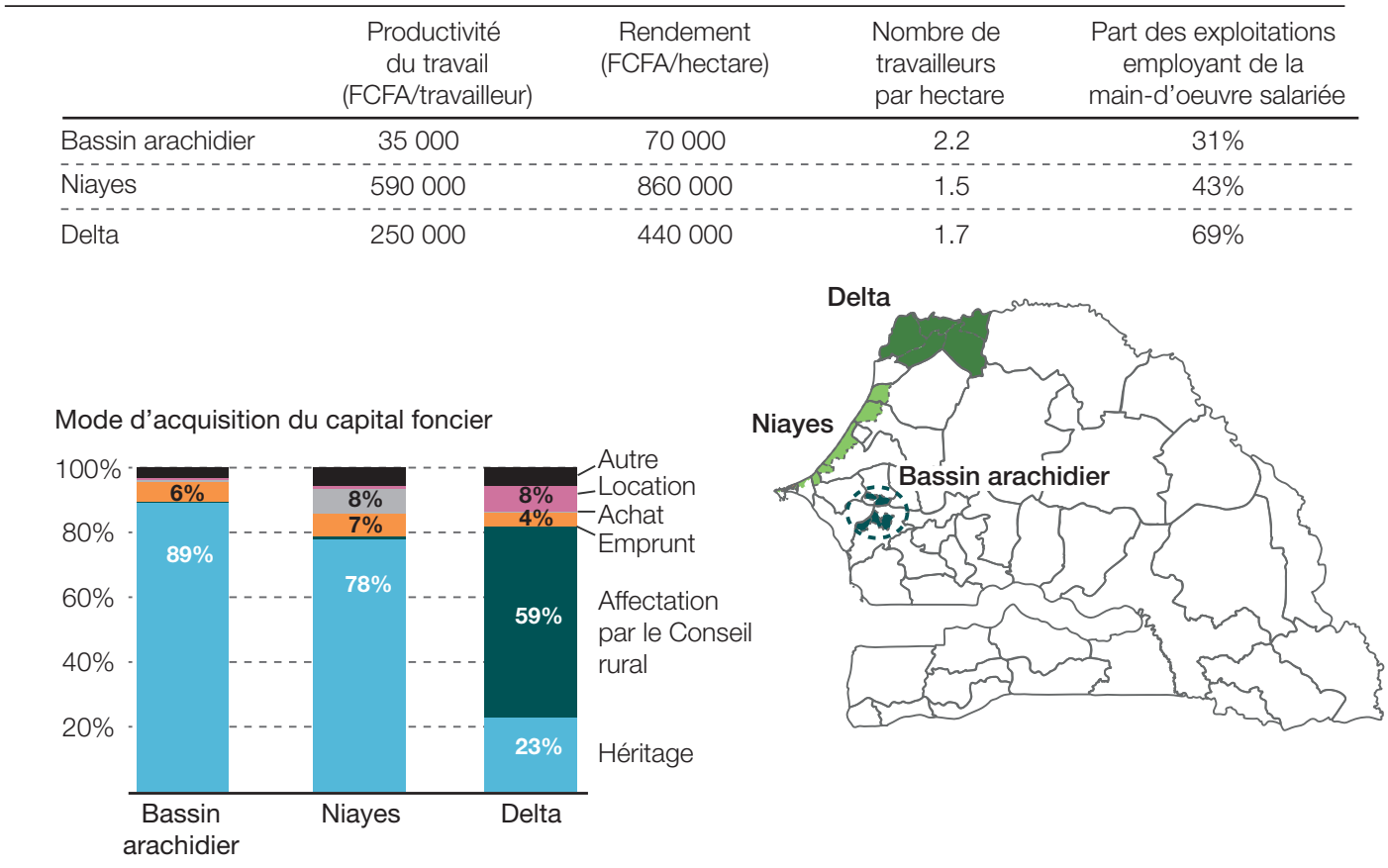

Note : franc de la Communauté financière d'Afrique (FCFA).

Source : calculs des auteurs à partir de IPAR (2015), « Emploi et migration des jeunes en Afrique de l'Ouest - Rapport final Sénégal ». 
L'analyse de l'emploi rural par sous-division administrative met en évidence une forte hétérogénéité spatiale en termes de diversification de l'emploi en milieu rural. Dans le sud de la Côte d'Ivoire et au Ghana, les zones rurales proches des grandes agglomérations urbaines sont plus diversifiées. Au Mali, les communes le long du fleuve Niger se caractérisent par une plus forte diversification de l'emploi. Au Nigéria, dans 99 des 299 districts administratifs couverts, l'emploi agricole représente moins de 50 \% de l'emploi total (carte 1).

Carte 1

Emploi rural non agricole dans l'économe alimentaire, par régions administratives

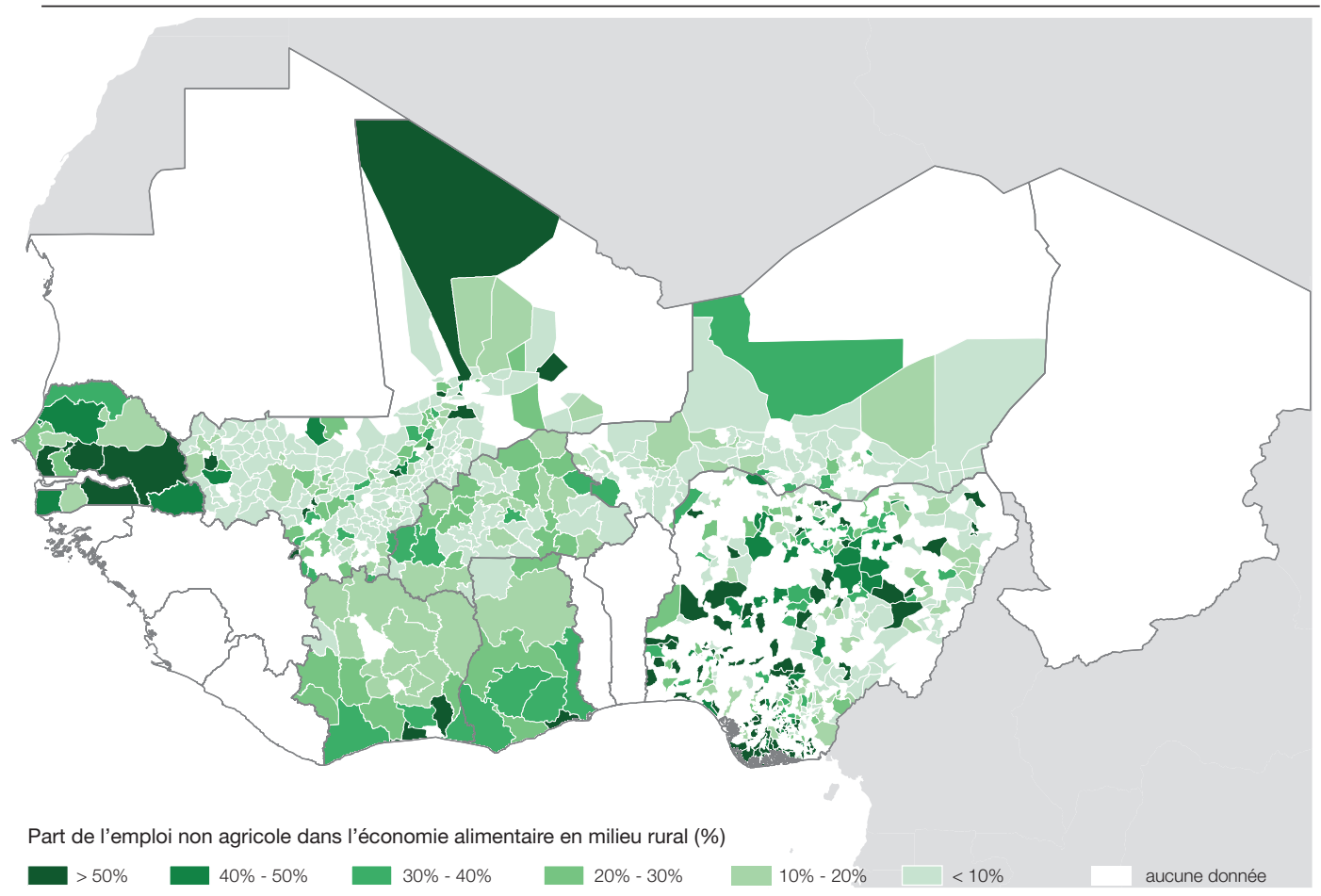

Sources : calculs des auteurs à partir de ANSD (2015), Enquête nationale sur l'emploi 2015 (base de données) ; Banque mondiale (2017a), Enquête sur la mesure des niveaux de vie (base de données) ; INS (2012), Enquête nationale sur la situation de l'emploi 2012, Côte d'Ivoire (base de données).

La proximité des marchés est importante pour le développement d'économies rurales diversifiées (Proctor et Berdegué, 2016 ; OCDE/CSAO, 2013 ; Dorosh et al., 2010). Les zones rurales bien reliées aux marchés d'intrants et d'extrants avec une production agricole spécialisée offrent plus d'opportunités de création de valeur ajoutée et d'emplois dans les segments aval des chaînes de valeur alimentaires. Les différences en termes d'accès aux infrastructures et aux services (centres de collecte, installations de transformation, de calibrage et de stockage, infrastructures de transport et capacités logistiques, structures de marché de gros et de détail, accès aux intrants et débouchés, y compris la terre et le capital) influent sur l'emploi local et les besoins en compétences. De récentes études microéconomiques corroborent l'incidence de la proximité des villes, des marchés et des infrastructures sur la part de l'emploi rural non agricole et les revenus (Jayne, Chamberlin et Headey, 2014) ${ }^{12}$.

Les villes petites et moyennes joueront un rôle important dans le développement de l'économie alimentaire. Elles représentent les points d'entrée des exploitations familiales dans l'économie alimentaire et consolident les activités et l'emploi non agricoles (Tacoli et Agergaard, 2017 ; Proctor et Berdegué, 2016). L'accès à ces opportunités est particulièrement important dans la promotion d'emplois décents et inclusifs (Christiaensen et Lawin, 2017). 
Le renforcement de liens en amont et en aval entre le milieu rural et les villes petites et moyennes est particulièrement important pour le développement des opportunités d'emploi en dehors des grandes villes (Henderson et McNamar, 2000 ; Proctor et Berdegué, 2016). Les progrès en matière de connectivité des zones rurales et urbaines grâce à la mise en place d'infrastructures, de services privés et publics de base (tels que l'éducation et la formation technique et professionnelle), de services de vulgarisation en agriculture et technologies alimentaires, de services de conseil et financiers, mais aussi d'infrastructures de marché et de transport efficaces dans les villes petites et moyennes sont au cœur de la croissance d'économies locales dynamiques et diversifiées (Proctor et Berdegué, 2016).

\section{IMPLICATIONS POLITIQUES}

L'économie alimentaire est le premier employeur de la région et continuera d'offrir de nombreuses opportunités avec l'accroissement de la population, de l'urbanisation et des revenus. L'agriculture représente la majorité des emplois de l'économie alimentaire, il importe donc d'en comprendre l'évolution. Il est possible d'améliorer la productivité agricole et les niveaux de vie des populations. Pour cela, il convient de déterminer quelles activités offrent les meilleures opportunités, de réduire les barrières à l'entrée pour les femmes et les jeunes, et d'anticiper la transition de l'emploi hors de l'agriculture. Ce dernier point est indissociable des enjeux liés à la disponibilité des terres et à la taille des exploitations, aux transformations rurales, à la diversification de l'emploi rural et aux changements structurels de manière plus générale.

Les activités non agricoles de l'économie alimentaire occupent une place particulièrement importante dans les stratégies d'emploi. Dans de nombreuses zones rurales, la plupart des emplois non agricoles sont dans l'économie alimentaire. Ces derniers y constituent une source majeure d'opportunités et de diversification des revenus. La création d'emplois non agricoles dépendra du soutien apporté aux entreprises privées, en particulier aux PME, au développement des chaînes de valeur et à l'acquisition de nouvelles compétences, ainsi que de l'amélioration du climat des affaires. Les femmes dominent dans les segments non agricoles, où elles représentent plus de $75 \%$ de l'emploi total. Les politiques et investissements qui promeuvent les activités non agricoles de l'économie alimentaire leur profiteront donc particulièrement. Les politiques agricoles et alimentaires ne prêtent pas encore suffisamment d'attention à ces activités.

Cette Note montre la forte hétérogénéité spatiale en Afrique de l’Ouest. Les zones rurales et rurales-urbaines ne sont pas toutes au même niveau de transformation de leur système alimentaire. Elles ne sont pas non plus confrontées aux mêmes contraintes pour assurer le développement d'économies locales diversifiées. Les migrations rurales-rurales et la mobilité au sens large devraient figurer parmi les instruments d'action privilégiés des stratégies pour l'emploi. Les analyses et les données détaillées disponibles sur le marché du travail, notamment sur les aspects spatiaux, font cependant généralement défaut. Ce manque de données empêche l'étude des dynamiques du marché du travail dans la région et limite la capacité des gouvernements et des responsables politiques à anticiper les transformations futures et à formuler des politiques d'emploi appropriées aux différents échelons locaux et nationaux. 


\section{NOTES}

1 Voir l'annexe 1 pour des informations sur les bases de données utilisées et la normalisation des codes d'activité.

2 Les auteurs remercient David Tschirley pour l'accès au programme Stata utilisé dans Tschirley et al. (2016).

3 Tschirley et al (2016) retiennent une définition plus large du système agroalimentaire, qui comprend la production, la transformation et le commerce de détail des matières premières agricoles non destinées à la consommation humaine, telles que le coton.

4 L'annexe 2 donne des précisions sur les modélisations.

5 L'une des raisons pourrait être que, dans le recensement de 2009, les chiffres sur l'urbanisation sont fondés sur deux définitions différentes de l'urbain.

6 La main-d'œuvre agricole participant à la production de coton est comptabilisée dans l'emploi agricole, mais exclue de l'emploi dans l'agriculture à destination de l'alimentation humaine, et ne fait pas partie du système alimentaire.

7 La part des importations alimentaires, estimée à $8 \%$, correspond à la part de la valeur des importations alimentaires extrarégionales dans les dépenses alimentaires totales des ménages. L'expression des importations alimentaires en pourcentage de la consommation alimentaire domestique souligne la diversité des produits alimentaires - allant de produits agricoles bruts ou légèrement transformés, comme le riz ou le blé, aux aliments hautement transformés, comme le poisson congelé ou les produits laitiers, qui incluent la valeur ajoutée hors agriculture - et de leur niveaux d'importation respectifs.

8 L'ETP correspond à 40 heures de travail par semaine pendant 52 semaines par an.

9 Le sous-emploi « visible » est défini comme une semaine de travail de moins de 40 heures.

10 À l'exclusion des boissons et de la restauration.

11 Les données présentées ici sont disponibles uniquement pour les sept pays pour lesquels des données détaillées sur l'emploi sont disponibles (Burkina Faso, Côte d'Ivoire, Ghana, Mali, Niger, Nigéria, Sénégal)

12 Les autres facteurs cités ayant une forte incidence sur la diversification des activités rurales non agricoles sont la productivité agricole, l'éducation, la taille du ménage et la taille de l'exploitation (Jayne, Chamberlin et Headey, 2014). 


\section{RÉFÉRENCES}

Allen, T. et P. Heinrigs (2016), « Les nouvelles opportunités de l'économie alimentaire ouest-africaine », Notes ouest-africaines, n¹, Éditions OCDE, Paris, http://dx.doi. org/10.1787/5jlwjg67l25f-fr.

Bouchama, N., et al. (2018), "Gender Inequality in West African Social Institutions», Notes ouest-africaines, $n^{\circ} 13$, Éditions OCDE, Paris, http://dx.doi.org/10.1787/fe5ea0ca-en.

Bricas, N., C. Tchamda et F. Mouton (2016), "L'Afrique à la conquête de son marché alimentaire intérieur. Enseignements de dix ans d'enquêtes auprès des ménages d'Afrique de l'Ouest, du Cameroun et du Tchad ", Études de l'AFD, n 12, Agence française de développement, Paris, http://www.afd.fr/.

Christiaensen, L. (2017), "Agriculture in Africa - Telling myths from facts: A synthesis", Food Policy, vol. 67, pp. 1-11, Elsevier, Amsterdam, https://doi.org/10.1016/j. foodpol.2017.02.002.

Christiaensen, L. et G. Lawin (2017), « L'emploi au sein de la transformation structurelle perspectives pour la Côte d'Ivoire ", in Emploi, Productivité et Inclusion pour Réduire la Pauvreté : Un diagnostic de la situation de l'emploi en Côte d'lvoire, Christiaensen, L. et P. Premand (dir. pub.), Banque mondiale, Washington DC, http://documents.worldbank.org.

Christiaensen, L. et P. Premand (dir. pub.) (2017), Emploi, productivité et inclusion pour réduire la pauvreté : Un diagnostic de la situation de l'emploi en Côte d'lvoire, Banque mondiale, Washington, DC, http://documents.worldbank.org.

Dorosh, P., H.G. Wang, L. You et E. Schmidt (2010), "Crop production and road connectivity in sub-Saharan Africa, A spatial analysis", Policy Research Working Paper, n 5385 , Région Afrique - Division développement durable, Banque mondiale, Washington DC, http://documents.worldbank.org/curated/en/319731468006253963/pdf/WPS5835.pdf.

Hebous, S. et T.T. Tran (2017), « Tendances et perspectives pour la création d'emplois formels en Côte d'Ivoire», in Emploi, productivité et inclusion pour réduire la pauvreté : Un diagnostic de la situation de l'emploi en Côte d'Ivoire, Christiaensen, L. et P. Premand (dir. pub.), Banque mondiale, Washington DC, http://documents.worldbank.org.

Henderson, J.R. et K.T. McNamara (2000), "The location of food manufacturing plant investments in Corn Belt counties", Journal of Agricultural and Resource Economics, vol. 25, n², pp. 680-697.

IPAR (2015), «Emploi et migration des jeunes en Afrique de l'Ouest - Rapport final Sénégal », Initiative prospective agricole et rurale, Dakar, http://www.ipar.sn.

Jayne, T.S., J. Chamberlin et D.D. Headey (2014), "Land Pressures, the evolution of farming systems, and development strategies in Africa: A synthesis", Food Policy, vol. 48, pp. 117, https://doi.org/10.1016/j.foodpol.2014.05.014

Kuznets, S. (1996), Modern Economic Growth: Rate, Structure and Spread, Yale University Press, New Haven, CT.

MADR/Côte d'Ivoire (2015), «Enquête-test des outils pour l'intégration de la dimension 'revenu des ménages' dans le suivi de la vulnérabilité en zone urbaine en Côte d'Ivoire : Cas du quartier Plateau de la Commune Attécoubé », Ministère de l'Agriculture et du Développement rural, Abidjan.

McCullough, E.B. (2017), "Labor productivity and employment gaps in Sub-Saharan Africa", Food Policy, vol. 67, Elsevier, http://dx.doi.org/10.1016/j.foodpol.2016.09.013.

Ministère de l'Alimentation et de l'Agriculture du Ghana (2016), "Pilot research-study on the integration of 'households income' in the monitoring of urban vulnerability in Ghana". Direction des statistiques, de la recherche et de l'information, Accra.

Nesheim, M.C., M. Oria et P.T. Yih (dir. pub.) (2015), "A Framework for Assessing Effects of the Food System", Committee on a Framework for Assessing the Health, Environmental, and Social Effects of the Food System, Food and Nutrition Board, Board on Agriculture and Natural Resources, Institute of Medicine, National Research Council, Washington, DC, National Academies Press, www.ncbi.nlm.nih.gov/books/NBK305168/.

OCDE/CSAO (à paraître), "L'urbanisation des pays de l'Afrique de l'Ouest 1950-2015 », Cahiers de l'Afrique de l'Ouest, Éditions OCDE, Paris.

OCDE/CSAO (2013), «Peuplement, marché et sécurité alimentaire », Cahiers de l'Afrique de l'Ouest, Éditions OCDE, Paris, http://dx.doi.org/10.1787/9789264187412-fr

OIT (2012a), Sectoral country profil - Brazil, Organisation internationale du travail, Genève, http://www.ilo.org.

OIT (2012b), Sectoral country profil - South Africa, Organisation internationale du travail, Genève, http://www.ilo.org.

Prieto Curiel, R., P. Heinrigs et I. Heo (2017), "Cities and Spatial Interactions in West Africa: A Clustering Analysis of the Local Interactions of Urban Agglomerations", Notes ouestafricaines, $n^{\circ}$ 5, Éditions OCDE, Paris, http://dx.doi.org/10.1787/57b30601-en.

Proctor, F.J. et J.A. Berdegué (2016), "Food systems at the rural-urban interface", Working Paper series, n¹94, Rimisp, Santiago, Chili. 
Reardon, T. (2015), "The hidden middle: the quiet revolution in the midstream of agrifood value chains in developing countries", Oxford Review of Economic Policy, vol. 31, $\mathrm{n}^{\circ}$ 1, pp. 45-63, https://doi.org/10.1093/oxrep/grv011.

Reardon, T. et al. (2015), "Urbanization, Diet Change, \& Transformation of the Downstream and Midstream of the Agrifood System: Effects on the Poor in Africa and Asia", Faith \& Economics, vol. 66, pp. 43-63, http://www.gordon.edu/ace/pdf/2015\%20Fall\%20-\%20 Reardon.pdf.

Staatz, J. et F. Hollinger (2016), "West African Food Systems and Changing Consumer Demands", Notes ouest-africaines, $n^{\circ}$ 4, Éditions OCDE, Paris, http://dx.doi.org/10.1787/ b165522b-en.

Tacoli, C. et J. Agergaard (2017), Urbanisation, rural transformations and food systems: the role of small towns, IIED, Londres, ISBN: 978-1-78431-418-7.

Tschirley, D., M. Kondo et J. Snyder (2016), "Downstream report", in "Agrifood Youth Employment and Engagement Study" (AGYEES), Allen, A., J. Howard, A. Jamison, T. Jayne, M. Kondo, J. Snyder, D. Tschirley et F. K. Yeboah (eds), Michigan State University, East Lansing, Michigan, http://www.isp.msu.edu.

\section{Bases de données}

ANSD (2015), Enquête nationale sur l'emploi 2015 (base de données), Agence nationale de la statistique et de la démographie, Sénégal, http://anads.ansd.sn/index.php/catalog/92 (consulté le 1er septembre 2017).

Banque mondiale (2017a), Enquête sur la mesure des niveaux de vie (LSMS), Banque Mondiale (base de données), http://microdata.worldbank.org/index.php/catalog/lsms (consulté le 1er septembre 2017).

Banque mondiale (2017b), Indicateurs de développement dans le monde (WDI) (base de données), Banque Mondiale, http://databank.banquemondiale.org/data/reports. aspx?source=indicateurs-du-d\%C3\%A9veloppement-dans-le-monde (consulté le 1er septembre 2017).

INS (2012), Enquête nationale sur la situation de l'emploi 2012, Institut national de la statistique, Côte d'Ivoire (base de données), http://www.ins.ci/n/nada/index.php/ catalog/78/get_microdata (consulté le 1er septembre 2017),

OIT (2017), Emploi par secteur, ILOSTAT (base de données), Organisation internationale du travail, http://www.ilo.org/ilostat/faces/oracle/webcenter/portalapp/pagehierarchy/ Page3.jspx?locale=fr\&_afrLoop=1901062925392086\#!\%40\%40\%3Flocale\%3Dfr\%26_ afrLoop\%3D1901062925392086\%26_adf.ctrl-state\%3D81g6pxny5_103 (consulté le 1er septembre 2017).

ONU (2014), Perspectives de l'urbanisation mondiale, révision 2014 (base de données), Division de la population, Organisation des Nations Unies, New York, https://esa.un.org/ unpd/wup/ (consulté le 1er septembre 2017). 


\section{ANNEXE 1 : SOURCES DES DONNÉES ET STANDARDISATION}

\section{Source des données}

D es données microéconomiques sur les ménages sont disponibles pour sept pays (Burkina Faso, Côte d’Ivoire, Ghana, Mali, Niger, Nigéria et Sénégal). Les bases de données de ménages des Enquêtes sur la mesure des niveaux de vie (LSMS) de la Banque mondiale (2017a) ${ }^{1}$ ont été utilisées dans cinq de ces pays (Burkina Faso, Ghana, Mali Niger et Nigéria). Plus précisément, les Enquêtes sur la mesure des niveaux de vie - Enquêtes intégrées sur l'agriculture (LSMS-ISA) ${ }^{2}$ ont été utilisées dans quatre pays (Burkina Faso, Mali, Niger et Nigéria). Pour la Côte d’Ivoire et le Sénégal, les estimations ont été réalisées à partir des dernières enquêtes disponibles sur l'emploi.

Détails et sources des sept bases de données utilisées :

Burkina Faso: Enquête multisectorielle continue 2014

http://microdata.worldbank.org/index.php/catalog/2538

Côte d'Ivoire: Enquête nationale sur la situation de l'emploi 2012

http://www.ins.ci/n/nada/index.php/catalog/78/get_microdata

Ghana: Ghana Living Standards Survey 6 (With Labour Force Module) 2012-2013

http://www.statsghana.gov.gh/nada/index.php/catalog/72

Mali: Enquête agricole de conjoncture intégrée 2014

http://microdata.worldbank.org/index.php/catalog/2583

Niger: Enquête nationale sur les conditions de vie des ménages et l'agriculture 2014

http://microdata.worldbank.org/index.php/catalog/2676

Nigéria: General Household Survey 2012-2013

http://microdata.worldbank.org/index.php/catalog/1952

Sénégal: Enquête nationale sur l'emploi 2015

http://anads.ansd.sn/index.php/catalog/92

\section{Standardisation}

Cinq classifications statistiques ${ }^{3}$ différentes des activités économiques sont utilisées dans les sept enquêtes. Des tableaux de conversion ont été établis pour convertir chaque classification dans une nomenclature commune. Ces tableaux de conversion sont disponibles sur demande.

Le niveau de désagrégation par activité - le plus détaillé, à trois ou quatre chiffres - est nécessaire pour identifier les activités du système alimentaire. Cette information n'est pas toujours disponible, en particulier pour le Nigéria, pays pour lequel on ne dispose que d'une classification des activités à deux chiffres. La méthode d'imputation de Tschirley et al (2017) a alors été appliquée pour estimer la répartition de l'emploi par segment du système alimentaire.

Cette méthode utilise les parts budgétaires des dépenses alimentaires des ménages, par zones rurales et urbaines, comme paramètres pour répartir l'emploi entre les activités de l'économie alimentaire. Par exemple, l’emploi dans le (c commerce de détail non spécifié » au Burkina Faso est réparti entre le commerce de détail alimentaire et non alimentaire en fonction, respectivement, des parts du budget alimentaire des ménages consacrées aux produits alimentaires et non alimentaires. Les données sur la consommation sont issues de la base de données sur la consommation mondiale de la Banque mondiale. 
Deux autres paramètres sont utilisés : i) la part de l'emploi agricole à destination de l'alimentation humaine au Nigéria, qui est reprise de Tschirley et al. (2017) ; et ii) la part de l'emploi dans les cultures de plantes à fibre (comme le coton) dans la production végétale totale en Côte d'Ivoire, qui est estimée à dires d'experts. Tous les paramètres utilisés sont disponibles sur demande.

\section{ANNEXE 2 : MODÉLISATION DE L’EMPLOI - DIX PAYS}

$\mathbf{L}$ 'emploi dans l'économie alimentaire est directement observé à partir de données d'enquête microéconomiques pour sept pays. Pour les dix pays restants (Bénin, Cabo Verde, Gambie, Guinée, Guinée-Bissau, Libéria, Mauritanie, Sierra Leone, Tchad et Togo), on ne dispose pas de données microéconomiques récentes. Une approche de modélisation en deux étapes a été mise en œuvre pour estimer la répartition de l'emploi dans les différents segments de l'économie agroalimentaire.

\section{Estimer l'emploi agricole dans l'économie alimentaire}

La première étape consiste à estimer l'emploi agricole dans l'économie alimentaire. La théorie économique et les études empiriques montrent qu'il existe une relation négative entre le niveau de PIB par habitant et la part de l'agriculture, aussi bien dans la valeur ajoutée totale que dans l'emploi (Kuznets, 1966). Ce phénomène est attribué à l'évolution rapide des nouvelles technologies agricoles, qui se traduit par une amélioration des rendements et de la productivité du travail, et au ralentissement de la croissance de la demande alimentaire par rapport aux autres biens et services. Selon la loi d’Engel, la part de l'alimentation dans les dépenses totales des ménages décroît à mesure que le revenu augmente. Les changements structurels dans la répartition des emplois par secteur sont en partie liés à cette relation négative entre croissance des revenus et part de l'alimentation dans le budget des ménages.

Un autre résultat empirique courant dans la littérature économique stipule qu'il existe une relation statistique positive entre la croissance des revenus et la part des dépenses consacrées aux produits alimentaires à plus haute valeur ajoutée. Selon la loi de Bennett, la composition du panier de produits alimentaires se transforme à mesure que le revenu augmente, avec une diminution de la part des féculents, compensée par une augmentation de la consommation de denrées à plus forte valeur ajoutée. Cette observation amène à considérer qu'il existe également une relation entre les revenus et la répartition de la valeur ajoutée et de l'emploi entre les différents segments de l'économie alimentaire.

On observe une forte corrélation entre le PIB par habitant et la part de l'emploi agricole dans l'économie alimentaire pour les sept pays (graphique A2.1). Ce résultat n'est pas suffisant pour poser l'existence d'une relation économique formelle entre ces deux variables, mais il indique l'existence d'une régularité statistique dans le contexte de l'Afrique de l'Ouest qui permet de formuler des hypothèses pour les dix pays pour lesquels on ne dispose pas de données détaillées sur l'emploi. On supposera donc dans le présent document que la part de l'emploi agricole dans l'économie alimentaire pour les dix pays pour lesquels on ne dispose pas de données suit la même relation que celle observée pour les sept autres pays.

\section{Estimer l'emploi non agricole dans l'économie alimentaire}

Dans une deuxième étape, on procède à l'estimation des parts de l'emploi dans les trois segments non agricoles de l'économie agroalimentaire. On observe qu'à l'exception de la Côte d'Ivoire, il existe une relation quasi-constante entre les segments de la transformation, de la commercialisation et de la restauration hors domicile (tableau A2.1), l'emploi dans la commercialisation et la restauration hors domicile représentant en moyenne quatre fois l'emploi dans le segment de la transformation. La simple moyenne arithmétique relevée pour six pays (à l'exclusion de la Côte d'Ivoire) sur ces trois segments est donc utilisée pour déterminer la répartition de l'emploi non agricole pour les dix pays restants. 
Graphique A2.1

Part de l'agriculture dans l'emploi de l'économie alimentaire et PIB par habitant

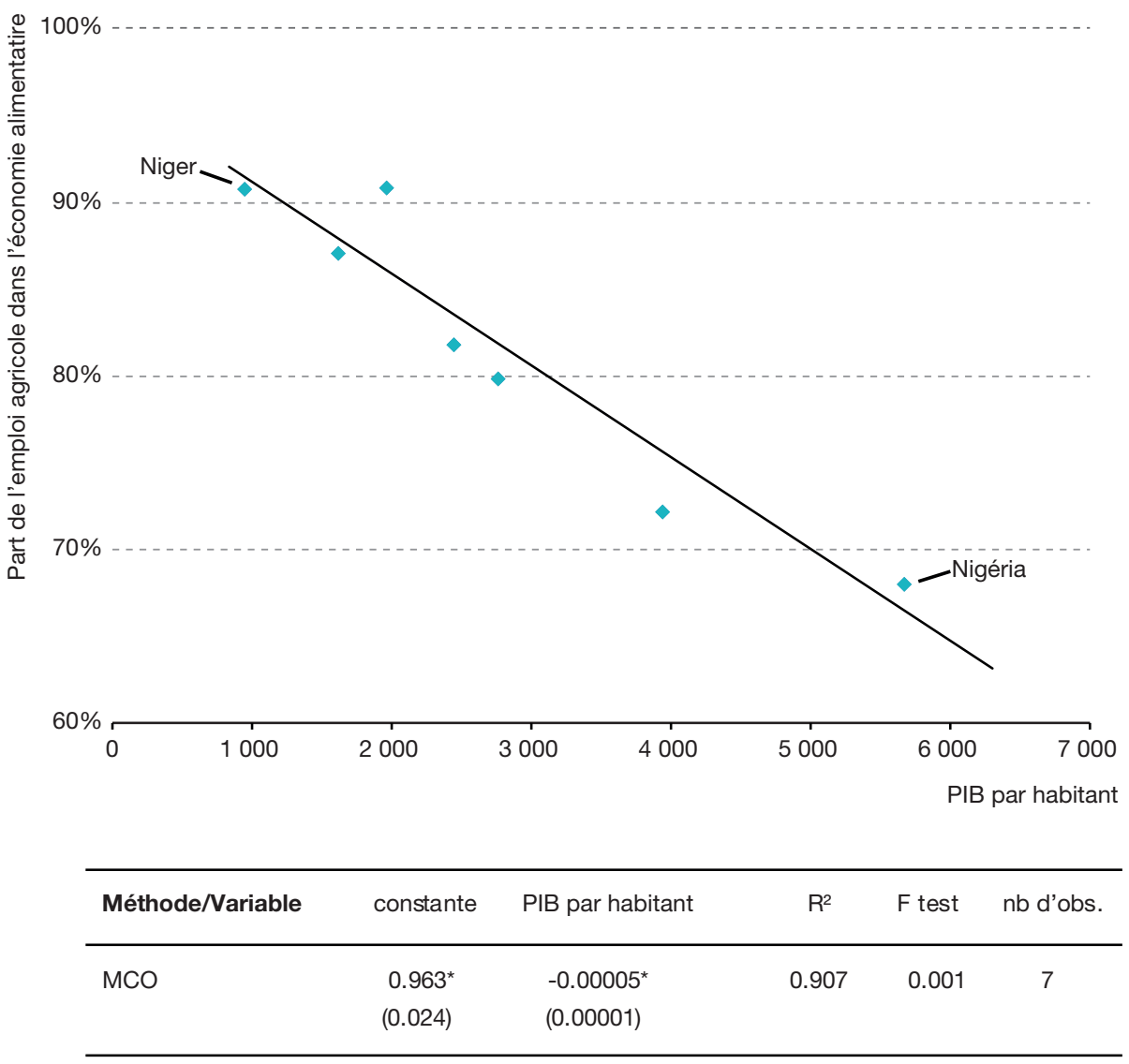

Note: la variable dépendante est la part de l'emploi agricole dans l'économie observée pour sept pays. Estimation par les moindres carrés ordinaires (MCO). Écarts-types entre parenthèses. Seuil de signification de $1 \%$ indiqué par*.

Sources: calculs des auteurs à partir de ANSD (2015), Enquête nationale sur l'emploi 2015 (base de données) ; Banque mondiale (2017a), Enquête sur la mesure des niveaux de vie (base de données) ; INS (2012), Enquête nationale sur la situation de l'emploi 2012, Côte d'Ivoire (base de données).

Tableau A2.1

Parts de l'emploi dans les segments non agricoles de l'économie alimentaire

\begin{tabular}{lccc} 
& $\begin{array}{c}\text { Transformation } \\
\text { alimentaire }\end{array}$ & $\begin{array}{c}\text { Commercialisation } \\
\text { et restauration } \\
\text { hors domicile }\end{array}$ & Ratio \\
\hline Burkina Faso & $2 \%$ & $11 \%$ & 5.0 \\
Côte d'Ivoire & $1 \%$ & $14 \%$ & - \\
Ghana & $6 \%$ & $22 \%$ & 3.7 \\
Mali & $2 \%$ & $7 \%$ & 4.6 \\
Niger & $3 \%$ & $7 \%$ & 2.5 \\
Nigéria & $7 \%$ & $25 \%$ & 3.3 \\
Sénégal & $3 \%$ & $17 \%$ & 5.3 \\
\hline & & Moyenne: & 4.1
\end{tabular}

Sources: calculs des auteurs à partir de ANSD (2015), Enquête nationale sur l'emploi 2015 (base de données) ; Banque mondiale (2017a), Enquête sur la mesure des niveaux de vie (base de données) ; INS (2012), Enquête nationale sur la situation de l'emploi 2012, Côte d'Ivoire (base de données). 
Enfin, les données de l'OIT sur l'emploi par secteur (primaire, secondaire et tertiaire) sont utilisées ${ }^{5}$ pour reconstituer les volumes d'emplois à partir des parts estimées précédemment. On note que l'emploi dans le segment agricole de l'économie alimentaire dans les sept pays représente entre 97 et $99 \%$ de l'emploi total du secteur primaire. La moyenne de ces parts est appliquée aux données de l'OIT pour estimer le nombre de personnes employées en agriculture dans l'économie alimentaire pour chacun des dix pays. Les données pour les autres segments sont récupérées de manière arithmétique à partir des parts estimées comme expliqué précédemment.

\section{NOTES}

1 Le programme LSMS de la Banque mondiale vise à produire des bases de données de qualité sur les ménages, à améliorer les méthodes de collecte de données et à renforcer les capacités des acteurs. http://econ.worldbank.org/WBSITE/EXTERNAL/EXTDEC/EXTRESEARCH/ EXTLSMS/0,,contentMDK:23506656 pagePK:64168445 piPK:64168309 theSitePK:3358997,00.html.

2 Le programme LSMS-ISA vise à encourager l'innovation et l'efficience dans les recherches statistiques sur les liens entre l'agriculture et la réduction de la pauvreté dans la région. http://econ.worldbank.org/WBSITE/EXTERNAL/EXTDEC/EXTRESEARCH/ EXTLSMS/O,,contentMDK:23512006 pagePK:64168445 piPK:64168309 theSitePK:3358997,00.html.

3 Classification internationale type, par industrie (CITI), révision 4 à 4 chiffres (Ghana) ; CITI, révision 4 à 2 chiffres (Nigéria) ; Première NAEMA, révision 1 (Sénégal) ; et nomenclature nationale (Burkina Faso, Mali et Niger).

4 Pour plus de renseignements, consulter : datatopics.worldbank.org/consumption/.

5 L'emploi dans les secteurs primaire, secondaire et tertiaire est basé sur la classification CITI. Le secteur primaire correspond à la section $\mathrm{A}(\mathrm{CITI}$ révision 4) ; il comprend l'agriculture, la chasse, la sylviculture et la pêche. Le secteur secondaire couvre les sections B-F (CITI révision 4) et comprend les activités extractives (y compris la production de pétrole), les activités de fabrication, la construction et la production/distribution de services publics (électricité, gaz et eau). Le secteur tertiaire correspond aux sections G-U (CITI révision 4) et il comprend le commerce de gros et de détail ainsi que les activités d'hébergement et de restauration; le transport, l'entreposage et les communications ; les activités financières et d'assurance, les activités immobilières et les activités de services administratifs et tous les autres services (éducation, santé, spectacles et autres services aux personnes). 
